\title{
Proceeding of the collaborated convention, the 20th Annual Meeting of the Japanese Society for Oral Mucous Membrane and the 21st Annual Meeting of the Japanese Society of Oral Pathology
}

Meeting Period: July 30-August 1, 2010

Meeting Venue: Osaka Dental University Hospital, Tenmabashi, Osaka, Japan (July 30) and

Osaka Dental University, Hirakata, Osaka, Japan (July 31-August 1)

Organizing Committee: Chairperson: Professor Shosuke Morita and Professor Akio Tanaka

First Department of Oral and Maxillofacial Surgery and Department of Oral Pathology,

Osaka Dental University, Kuzuhahanazono-cho, Hirakata-shi, Osaka, Japan

\section{Program}

Opening Address: S. Morita and A. Tanaka (Osaka Dental University)

Special Lecture I:

Speaker: T. Itoh (Kobe University)

Special Lecture II:

Speaker: T. Hashimoto (Kurume University)

Scientific Symposium:

Symposium I: Toward Establishment of the Clinical Guideline of Oral Lichen Planus

Speaker; T. Fujibayashi (Kanagawa Dental College), Y. Sugawara (Tohoku University),

Y. Jinbu (Jichi Medical University), D. Ito (Tokyo Medical and Dental University),

S. Nakamura (Kyushu University), K. Komiyama (Nihon University),

A. Tanaka (Osaka Dental University), T. Saku (Niigata University),

H. Maeda (Aichi-Gakuin University), H. Hasegawa (Matsumoto Dental University)

Symposium II: Pathological Diagnostic Pitfall in the Oral Region

Speaker; T. Hirose (Tokushima Prefectural Central Hospital),

T. Yoshino (Okayama University)

Specific Report: Classification of the oral leukoplakia

Speaker: D. Ito (Japanese Society for Oral Mucous Membrane)

Case Discussion: 13 Titles

General Session: 62 Titles

Poster presentation: 37 Titles

Slide Seminar:

1. Autopsy: S. Hara (Kobe University)

2. Salivary grand: H. Harada (Sakai Municipal Hospital)

3. Cytology: Y. Tanaka (Tokyo Dental College)

\begin{abstract}
s
Symposium I: Toward establishment of the clinical guideline of oral lichen planus

Fujibayashi $T^{1}$, Sugawara $\mathrm{Y}^{1}$, Jinbu $\mathrm{Y}^{1}$, Ito $\mathrm{D}^{1}$, Nakamura $S^{1}$, Komiyama $K^{2}$, Tanaka $A^{2}$, Saku $T^{2}$, Maeda $\mathrm{H}^{2}$, Hasegawa $\mathrm{H}^{2}$

${ }^{1}$ Japanese Society for Oral Mucous Membrane. and ${ }^{2}$ The Japanese Society of Oral Pathology

Oral lichen planus (OLP) is a common disorder in the oral cavity. In 2009, it was agreed between JSOMM and JSOP to make a collaborative working project for "The Standard Guideline for the Clinical Management of OLP". The working group (the OLP committee) started the first
\end{abstract}

year project by analyzing clinically typical OLP lesions with reticular pattern in the bilateral buccal mucosa. A total of 154 cases of such OLP specimens were submitted to the committee from 34 facilities across the country and the committee started to analyze them. The interim report of the work will be presented at this symposium. The committee would like to share common understandings on various issues of current OLP diagnosis in Japan through discussion with both societies' members. 


\section{Symposium II: Pathological diagnostic pitfall in the oral region}

\section{Soft-tissue tumors of the head and neck region Hirose $\mathrm{T}$ \\ Department of Diagnostic Pathology, Tokushima Prefectural Central Hospital}

Soft-tissue tumors are groups of varied mesenchymal and neuroectodermal tumors including adipocytic, fibroblastic/myofibroblastic, so-called fibrohistiocytic, smooth muscle, pericytic, skeletal muscle, vascular, chondroosseous, peripheral nerve and uncertain differentiation tumors. The head and neck region as well as the extremities and trunk are common sites where soft tissue tumors affect. Several soft tissue tumors tend to arise in the head and neck region, as follows. Spindle cell/pleomorphic lipoma occurs in the posterior neck. Among fibrblastic/myofibroblastic tumors, cranial fasciitis, myofibroma/myofibromatosis, and low-grade myofibroblastic sarcoma often affect the head and neck. Embryonal and alveolar rhabdomyosarcoma also arises in the orbit, pharynx, middle ear, auditory canal, nasal cavity and paranasal sinus. Epithelioid hemangioma and lymphangioma are common vascular tumors in the head and neck. Granular cell tumor and alveolar soft-part sarcoma frequently occur in the tongue. Occasionally, synovial sarcoma also affects the head and neck. The diagnoses of soft-tissue tumors may sometimes be difficult, in that the differential diagnoses often include many heterogeneous tumor entities that show morphologic similarities. Therefore, in addition to microscopic characteristics, clinical features and radiological information are mandatory for the differential diagnosis. Furthermore, appropriate immunohistochemical approaches and molecular cytogenetic analyses are often required for the diagnosis of soft-tissue tumors.

\section{Malignant lymphoma of the head and neck: including diagnostic pitfalls}

Yoshino T

Department of Pathology, Okayama University, Graduate School of Medical, Dentistry and Pharmaceutical Sciences

Malignant lymphomas frequently arise from head and neck organs, including lymph nodes and extranodal organs. Almost all Hodgkin lymphomas are nodal origin. Extranodal organs can be subdivided into three groups. The oral cavity, pharynx and paranasal cavities are diffuse large B-cell lymphoma (DLBCL)-tropic. The mucosa-associated lymphoid tissue (MALT) lymphomas are frequently found in the salivary glands and thyroid gland. NK/T-cell lymphomas, which is Asian-tropic and associated with EB virus, are often found not only nasal cavity but pharynx and larynx. To differentiation of these three lymphomas is crucial for choosing therapeutic ways and for quite different clinical outcomes. Another important point for making diagnosis of lymphomas is differential diagnosis. As a matter of fact, only approximately $70 \%$ of clinically suspected lymphoma cases were "true" lymphomas, in other words, 30\% such cases were not lymphoma, but other benign or malignant tumors or other non-neoplastic lesions. It may be more important than subclassification of lymphomas. IgG4-related disease has recently been recognized as a unique lymphoproliferative disorders which has probably often been erroneously diagnosed as lymphomas. In the present paper, I would like to show why we should make diagnosis of lymphomas correctly by using immunohistochemical and/or immunogenotypical methods. And also, what kind of diseases or lesions are included for differential diagnosis of lymphomas and the pitfalls, especially.

Specific Report: Classification of the oral leukoplakia Ito $\mathrm{D}^{1}$, Maeda $\mathrm{H}^{1}$, Kawabe $\mathrm{R}^{1}$, Kitagawa $\mathrm{Y}^{1}$, Kusama $\mathrm{M}^{1}$, Jinbu $\mathrm{Y}^{1}$, Nagumo $\mathrm{M}^{1}$, Takashi $\mathrm{F}^{1}$, Mataga $\mathrm{I}^{1}$, Morimoto $\mathrm{M}^{1}$, Yamane $\mathrm{G}^{1}$, Yamamoto $\mathrm{T}^{1}$, Nakamura $\mathrm{S}^{1}$

${ }^{1}$ Committee for Terminology and Classification, Japanese Society for Oral Mucous Membrane

The Committee for Terminology and Classification, JSOMM, had started to develop a classification of oral leukoplakia in 2005, and the draft has been accepted by the JAOMM directors in 2009. Here we present the classification with some typical cases.

The leukoplakia was classified to two definitive clinical types, "homogeneous" and "non-homogeneous". Some frequent clinical types of the former were listed, and the latter was additionally classified to three subtypes, nodular, verrucous, and speckled.

The degree of the keratinization and epithelial dysplasia was asked to be clearly noted on the histopathologic diagnosis.

The differential diagnoses were listed as "the related lesions/diseases".

\section{Case Discussion}

\section{B-01. Mandibular tumor}

Ito H, Yuuko Sakurai Y, Sugiura J, Okuyama N, Kato M Division of Oral Pathology, Department of Oral Medical Science, Ohu University School of Dentistry

A 34-year-old woman was admitted to a hospital with a chief complaint of swelling at anterior part of her mandible. Radiographically, from right to left cuspids, a destructive radiolucent lesion associated with root resorption of the anterior teeth was observed. Histopathologically, bulk of lesion consisted of cords, nests or sheets of epithelioid cells, which had large, round nuclei with prominent nucleoli. The stroma was collagenous and hyaline. Vimentin, S-100 protein, and GFAP were detected in the tumor cells by inmmunohisitocemistry. However, keratin, p63, and $\alpha$ smooth muscle actin were negative. Ultrastructurally, desmosomes were not observed in the tumor cells. 
1B-02. Tumor of the mandible

Cheng $\mathrm{J}^{1}$, Tsuneki $\mathrm{M}^{1}$, Maruyama $\mathrm{S}^{1,4}$, Yamazaki $\mathrm{M}^{1}$, Kanamaru $\mathrm{S}^{2}$, Shingaki $\mathrm{S}^{2}$, Saito $\mathrm{C}^{2}$, Nishiyama $\mathrm{H}^{3}$, Hayashi $\mathrm{T}^{3}$, Saku $\mathrm{T}^{1}$

${ }^{4}$ Divisions of ${ }^{1}$ Oral Pathology, ${ }^{2}$ Reconstructive Surgery for Oral \& Maxillofacial Region, ${ }^{3}$ Oral \& Maxillofacial Radiology, Niigata University Graduate School of Medical \& Dental Sciences, ${ }^{4}$ Oral Pathology Section, Department of Surgical Pathology, Niigata University Hospital

A 35-year-old female have had a diffuse swelling in her mandible for 22 years. It was diagnosed twice by biopsies as fibrous dysplasia in 1990 and 1999. In 2009, image analyses including CT revealed an intraosseous enhancing expansile mass lesion with sporadic irregular-shaped calcified materials in the submental region, in addition to the longstanding diffuse radiopaque lesion expanding to the ramus portion. Under a clinical diagnosis of malignant mesenchymal tumor arising in the background of fibrous dysplasia, it was surgically removed. Histopathologically, the lesion involved the inside of the mandible extensively and expanded towards the surrounding soft tissue. It was composed of a dense proliferation of fibrous tissues scattering immature irregularshaped boney trabeculae.

\section{B-03. A case of mandibular tumor}

Yada N, Kashima K, Daa T, Yokoyama S

Department of Diagnostic Pathology, Faculty of Medicine, Oita University

Patient was a 76-year-old female who had an occlusal pain for one mouth in her right molar region of the mandible. Panoramic radiograph showed a radiolucent mass lesion with irregular margin and an impacted tooth in the right mandible. Macroscopically, the resected specimen showed a whitish-yellow solid tumor in the mandible with focal invasion to the gingiva. Histologically, the tumor was composed of various-sized, solid or cystic nests. Most tumor cells were glycogen-rich clear cells, but basophilic cells were focally observed. Nuclear atypia was slight with occasional mitotic figures (8/10 HPF). Immunohistochemically, labeling indices of Ki-67 and PCNA were $9.9 \%$ and $36.8 \%$, respectively.

\section{B-04. Tumor of the upper lip}

Tsuneki $\mathrm{M}^{1}$, Cheng $\mathrm{J}^{1}$, Maruyama $\mathrm{S}^{1,4}$, Yamazaki $\mathrm{M}^{1}$, Oda $\mathrm{Y}^{2}$, Shingaki $\mathrm{S}^{2}$, Saito $\mathrm{C}^{2}$, Nishiyama $\mathrm{H}^{3}$, Hayashi $\mathrm{T}^{3}$, Saku $\mathrm{T}^{1,4}$

Divisions of ${ }^{1}$ Oral Pathology, ${ }^{2}$ Reconstructive Surgery for Oral \& Maxillofacial Region, and ${ }^{3}$ Oral and Maxillofacial Radiology, Niigata University Graduate School of Medical \& Dental Sciences; ${ }^{4}$ Oral Pathology Section, Department of Surgical Pathology, Niigata University Hospital

A 70-year-old male patient had a painless swelling in his right side of the upper lip. After the diagnostic confirmation of a salivary gland tumor by clinical examinations including image analyses and biopsy, the tumor was surgically removed. Histopathologically, the removed tumor, located between the submucosal layer of the buccal mucosa and the buccal subcutis, was relatively well demarcated with some foci invading surrounding tissues. The tumor was basically composed of a papillary proliferation of single layered mucous cells with obvious cellular and structural atypia, which formed many various-sized cystic cavities.

\section{B-05. Granulomatous lesion of the maxilla}

Fujita $S^{1}$, Yamada $S^{2}$, Kawasaki $G^{2}$, Ikeda $T^{1}$

Department of Oral Pathology and Bone Metabolism, Unit of Basic Medical Sciences ${ }^{1}$, Department of Oral and Maxillofacial Surgery, Unit of Translational Medicine ${ }^{2}$, Course of Medical and Dental Sciences, Nagasaki University Graduate School of Biomedical Sciences

A 58-year old male was referred to Nagasaki University Hospital for farther examination of pain of the right temporomandibular joint and trismus. Panoramic radiography and CT revealed irregular radiolucent area in the right side of the maxilla. After the administration of the antiphlogistics, partial maxillectomy was performed. Removed specimen showed granulation tissue including inflammatory round cells and bone spiculae. Multinucleated giant cells were intermingled in the granulation tissue, and adhered to the resorbed bone fragments. These giant cells had palisading nuclei along the cell membrane. They were accompanied with accumulation of histiocytes. Small foci of necrotic tissue were also noticed. Ziehl Neelsen stain revealed no acid-fast bacteria.

\section{B-06. A parotid lesion}

Ehara M, Aoki R, Nakashima A

Saiseikai Fukuoka General Hospital, Division of Pathology

A 53-year-old female visited our hospital because of the swelling at the right auricular and supraauricular region. The lesion was diagnosed as a benign parotid gland tumor by MRI, and was resected. Macroscopically, removed mass, measuring $2.7 \times 1.9 \mathrm{~cm}$, showed grayish white multinodular mass, to which normal parotid gland was partially intermingled. Histopathologically, the mass was made up of proliferation of double layered ductal structures, forming nests of varying size to multiple nodules. Immunohistochemically, the outer layered cells were positive for $\alpha$-SMA. Some of the proliferating cells have fine to extra-fine PASpositive granules in the cytoplasm.

\section{B-13. Tumor of the neck: a case investigation}

\section{Harada $\mathrm{H}$}

Department of Pathology and Research, Sakai Municipal Hospital

A case of neck tumor arising in a 59-year-old male is presented. The patient had a history of sensory disturbance of the right neck followed by a slowly growing mass of the same location, which revealed destruction of C3-C5 vertebral arch in CT imaging. Open biopsy failed to establish histological diagnosis and no primary tumor could be found in any other general sites. The patient underwent surgical resection of the tumor, radical neck dissection and postoperative radiation of 50Gy doze. Histologically, the tumor was characterized by "granuloma-like pattern" and immunohistochemical reactivity for CD34 in addition to 
epithelial and myogenic markers.

\section{B-14. A case of the right parapharyngeal tumor Kusafuka K, Nakajima T \\ Pathology Division, Shizuoka Cancer Center Hospital and Research Institute}

The patient was 50-year-old Japanese woman, who noticed the right pharyngeal swelling and dysphagia. CT scan showed soft tissue lesion with micro-calcification in the right parapharyngeal space and low to mildly high intensity. MRI showed relatively well-defined solid mass. The tumor resection was performed. Grossly, the tumor was a $62 \times 40 \times$ $30 \mathrm{~mm}$-sized, brownish, polycystic, which contained bloody material. Histologically, the tumor consisted of the cavernous / dilated spaces, and slit-like structures with spindle cells, which showed mildly atypia. Fibrin or organized thrombi and hemosiderin deposition were seen, whereas microcalcification was seen. Partly, papillary growth or vacuolated epithelioid cells were also observed.

\section{B-15. A case of gingival tumor}

Matsumoto $\mathrm{N}^{1}$, Mukae $\mathrm{S}^{1}$, Omagari $\mathrm{D}^{1}$, Asano $\mathrm{M}^{1}$, Komiyama $\mathrm{K}^{1}$

1Department of pathology, Nihon University School of Dentistry

A 58-year-old male was referred to our hospital due to rapidly growing solid gingival mass at mandibula. At his first visit, a mass, measuring $26 \times 21 \mathrm{~mm}$ in size, was protruded from the right molar area. Clinical examinations including CT and MRI revealed no apparent bone resorption. Palpable lymph node was found in right submandibular area. Excisional biopsy was carried out. Histologically, the tumor was covered with necrotic tissues and was consisted of large, polygonal-shaped and highly atypical cells proliferation. Immunohistochemically, the tumor cells were positive for vimentin, NSE, and synaptophysin, while negative for CD99, high- and low-molecular weight keratins, EMA, and panel of melanocytic, lymphocytic and muscular markers. After the excision, swelling of supraclavicular lymph node and large nodular lesion in the apex of lung were presented. Then in 7 months, the patient died of respiratory failure.

\section{B-16. Palatal tumor}

Yoshida $\mathrm{M}^{1}$, Ogawa $\mathrm{I}^{2}$, Ohbayashi $\mathrm{M}^{1,2}$, Kudo $\mathrm{Y}^{1}$, Miyauchi $\mathrm{M}^{1}$, Takesue $\mathrm{N}^{3}$, Sasaki $\mathrm{N}^{4}$, Takata $\mathrm{T}^{1}$

${ }^{1}$ Department of Oral and Maxillofacial Pathobiology, Graduate School of Biomedical Sciences, Hiroshima University, ${ }^{2}$ Center of Oral Clinical Examination, Hiroshima University Hospital, ${ }^{3}$ Oral and Maxillofacial Surgery, Hiroshima University Hospital and ${ }^{4}$ Department of Pathology, Kure Kyosai Hospital

A 75-year-old female, who had been diagnosed as Sjogren syndrome by ophthalmologist 20 years before, noticed a nontender swelling in left side of the palate for about 4 months. Physical examination revealed a sessile mass, measuring $22 \times 14 \mathrm{~mm}$, covered with normal mucosa. After the biopsy based on the clinical diagnosis of salivary gland tumor, it was resected with surrounding soft tissue. The resected tumor was solid and relatively ill-defined with light brown to yellowish-white color. Histopathologically, the palatal gland is widely replaced by small or intermediatesized lymphocytic cells with lymphoid follicles. The remaining ducts are frequently infiltrated by these cells. The lymphocytic cells are mainly positive for CD20 and bcl-2. Plasma cells are also intermixed with these cells.

\section{B-17. A case of palatal tumor}

Urano M, Mizoguchi Y, Kuroda M

Department of Diagnostic Pathology, Fujita Health University, School of Medicine

A case of palatal tumor of a-64-year-old female patient. The tumor was $20 \times 10 \mathrm{~mm}$ in size. Microscopically, in the submucosal lesion of the palate, the tumor invaded into the surrounding minor salivary glands without capsule formation. The tumor had mainly low to high papillary components and cribriform formation, and composed of columnar cells with mucin, arranged in a small multicystic pattern. Cytological atypia were moderate. Necrosis and mitotic figures were often seen. Vascular and perineural invasion were not evident. Mucous elements were observed in a hyalinized stroma accompanying with histiocytes reaction and cholesterin clefts.

\section{B-18. Tumor of the palate}

Katase N, Tamamura R, Fujii M, Tsujigiwa H, Mano T, Ueyama Y, Nagatsuka H

${ }^{1}$ Department of Oral pathology and medicine, Graduate School of Medicine, Dentistry and Pharmaceutical Sciences, Okayama University

${ }^{2}$ Department of Oral and Maxillofacial Surgery, Yamaguchi University Graduate School of Medicine

A male patient in his 70's referred to the hospital, complaining the bleeding from tumor-like mass at the palate. The lesion was $38 \times 22 \mathrm{~mm}$ in size, with rough and erosive surface. CT and MRI revealed lymph nodal metastasis in the neck. With the clinical diagnosis of malignant tumor, biopsy followed by surgical resection of the tumor and neck dissection was performed. Histologically, the tumor cells consisted from polygonal squamous cells and small basal cell like cells. In the tumor nest, keratinization, comedo-like necrosis and duct-like structure with abundant basement membrane materials were observed, which showed similar appearance to salivary gland tumor.

\section{B-19. A case of serendipitously-detected maxilla lesion at orthognathic surgery}

Usami $\mathrm{Y}^{1,2}$, Takenobu $\mathrm{T}^{3}$, Kishino $\mathrm{M}^{4}$, Sato $\mathrm{S}^{4}$, Ishida $\mathrm{K}^{4}$, Ogawa $\mathrm{Y}^{4}$, Toyosawa $\mathrm{S}^{4}$, Fukuda $\mathrm{Y}^{1}$

${ }^{1}$ Clinical Laboratory, Osaka University Dental Hospital

${ }^{2}$ Division of Pathology, Kobe City Medical Center General Hospital

${ }^{3}$ Department of Oral and Maxillofacial Surgery, Kobe City Medical Center General Hospital

${ }^{4}$ Department of Oral Pathology, Osaka University Graduate School of Dentistry

Because of skeletal open bite, a 19-year-old man who was scheduled for orthognathic surgery, was referred to our hospital. Intraoral examination shows multiple small nodular 
lesion found in oral mucosa. Le Fort I osteotomy and bilateral intraoral vertical ramus osteotomy was performed. The excised bone tissue of the maxilla and mucosal nodule were examined.

Histologically, bone-marrow space of the jaw bone was composed of fatty marrow, and uncapsulated aggregation or proliferation of peripheral nerves were observed. The aggregation of peripheral nerves were also seen in submucosal tissue of the mucosal lesion.

\section{General Session}

1A-01. The relation between oral mucosal disease and smoking or drinking habits

Sasaoka K ${ }^{1}$, Susa $\mathrm{T}^{1}$, Kanno $\mathrm{K}^{1}$, Negishi $\mathrm{A}^{1}$, Yokoo $\mathrm{S}^{1}$ ${ }^{1}$ Department of Stomatology and Oral Surgery Gunma University Graduate School of Medicine

A questionnaire to examine the relation between oral mucosal disease and smoking and/or drinking was put to 336 patients (20 years old or older) who had consulted our department. The risk of the disease was significantly higher in men (not women) who smoked or drank compared with those who did not. The odds ratio increased 12.38 times in men who both smoked and drank compared with those who did neither.

1A-02. Development of computer supported diagnostic system of oral mucous disease

Ohtani $\mathrm{T}^{1}$, Habu $\mathrm{M}^{1}$, Horio $\mathrm{K}^{2}$, Yamakawa $\mathrm{T}^{2}$, Tominaga $\mathrm{K}^{1}$

${ }^{1}$ Division of Maxillofacial Diagnostic and Surgical Science, Department of Oral and Maxillofacial Surgery, Kyushu Dental College.

${ }^{2}$ Graduate School of Life Science and Systems Engineering, Kyushu Institute of Technology

Oral mucous disease normally detected during routine oral examination. They are visually distinct. Therefore, the specialist can diagnose probably by observation only. However, it is difficult for general dentist to diagnose those lesions. Then, we thought that introducing computer supported diagnostic system of oral mucous disease should make diagnosis easier for them. Fundamental theory of computing was not directed till date. So we use "soft computing" this time. At present, the white patches of oral lichen planus is being extracted from digital images, which can be compared with other oral mucous lesions.

1A-03. Acetic acid treatment for wrinkle free oral mucosal epithelia in paraffin section preparation Shahidul A $\mathrm{M}^{1}$, Maruyama $\mathrm{S}^{2}$, Cheng $\mathrm{J}^{1}$, Al-Eryani $\mathrm{K}^{1}$, Yamazaki $\mathbf{M}^{1}$, Hasegawa $\mathrm{M}^{1,3}$, Tsuneki $\mathbf{M}^{1}$, Saku $\mathrm{T}^{1,2}$ Divisions of ${ }^{1}$ Oral Pathology, ${ }^{3}$ Reconstructive Surgery for Oral and Maxillofacial Region, Niigata University Graduate School of Medical \& Dental Sciences, ${ }^{2}$ Oral Pathology Section, Department of Surgical Pathology, Niigata University Hospital

For histopathological assessment of oral borderline malignancies, it is important to carefully detect subtle epithelial changes on fully stretched tissue sections. However, it is not generally easy to obtain wrinkle-free sections when using formalin-fixed paraffin-embedded oral mucosal samples. Since acetic acid treatment is already utilized for large brain tissue sections, we examined whether that treatment was also effective for oral mucosal tissues containing normal to malignant epithelial lesions. Paraffin sections were floated in various concentrations of acetic acid for 10 minutes after stretching in water for 1 minute, then wrinkle formations were examined using hematoxylin and eosin staining, as well as for staining intensity with keratin immunohistochemistry. Wrinkles were formed in both epithelial and connective tissue zones of sections treated with less than a $40-\mathrm{mM}(0.25 \%)$ concentration of acetic acid. In contrast, treatments with concentrations at $80 \mathrm{mM}$ $(0.5 \%)$ and higher resulted in cracking between the epithelial layer andlaminapropria, as wellas poorimmunohistochemical results for keratins 13 and 17 , even though the wrinkles completely disappeared. These results indicate that $40 \mathrm{mM}$ is the optimal concentration of acetic acid solution to prevent wrinkles in the epithelial layer while maintaining the immunohistochemical qualities of oral mucosa tissue sections, especially those containing borderline malignant epithelial lesions.

1A-04. Study of the form of the intrapapillary capillary loops of oral mucosa in healthy volunteers Sakamaki $\mathrm{H}^{1}$, Kondoh $\mathrm{T}^{2}$, Suzuka $\mathrm{Y}^{1}$, Okawa $\mathrm{Y}^{1}$, Hosaka $\mathrm{M}^{1}$, Aso $\mathrm{T}^{1}$, Asaga $\mathrm{T}^{1}$, Ishida $\mathrm{Y}^{1}$, Yoshida $\mathrm{N}^{1}$, Kusaka $\mathrm{K}^{1}$, Matsui $\mathrm{K}^{1}$

${ }^{1}$ Department of Dental Hygiene, Faculty of Health Sciences, Chiba Prefectural University of Health Sciences

${ }^{2}$ Department of Maxillofacial Surgery, Nihon University School of Dentistry at Matsudo

We examined intrapapillary capillary loops (IPCLs) in the oral mucosa of 37 healthy female volunteers (mean age, 20.5 years) using contact mucoscopy (CMUS). IPCL type in the marginal gingiva of mandibular medial incisor was classified into 2 types: the same as perionychium (82.7\%); and obliquely running vessels (18.3\%). IPCL was frequently observed at the tongue verge. IPCLs were arranged regularly in the buccal mucosa of 36 subjects (97.3\%). CMUS was considered useful for diagnosing oral mucosal lesions by classification of the IPCL in normal oral mucosa.

\section{A-05. Expression of melatonin 1a receptor in rat oral mucosa}

Umeki $\mathrm{H}^{1}$, Shimozuma $\mathrm{M}^{1}$, Mishima $\mathrm{K}^{2}$, Tokuyama $\mathrm{R}^{1}$, Tatehara $\mathrm{S}^{1}$, Ide $\mathrm{S}^{1}$, Saito $\mathrm{I}^{2}$, Satomura $\mathrm{K}^{1}$

${ }^{1}$ Second Department of Oral and Maxillofacial Surgery, Department of Oral Medicine and Stomatology, School of Dental Medicine, Tsurumi University

${ }^{2}$ Department of Pathology, School of Dental Medicine, Tsurumi University

Melatonin, a hormone secreted by a pineal gland mainly at night, is involved in various physiological processes such as control of circadian rhythms. Recently, melatonin was proven to exist in saliva. This suggests that melatonin may 
possess a physiological role in oral mucosa. In this study, to examine the possibility that oral mucosal cells are target of melatonin, the expression of melatonin 1a receptor (Mel1aR) in rat oral mucosa was immunohistochemically investigated. As a result, prickle and granular cells were positive for MellaR. This result suggested that melatonin plays a physiological role in some functions of oral mucosa.

1A-06. Expression and function of retinoic acidinducible gene-I (RIG-I) in oral keratinocyte and fibroblast

Fukui A, Ohta K, Shigeishi H, Nishi H, Takechi M, Kamata N

Department of Oral and Maxillofacial Surgery, Division of Cervico - Gnathostmatology, Programsfor Applied Biomedicine, Graduate School of Biomedical Sciences, Hiroshima University

The retinoic acid-inducible gene I product (RIG-I) has been identified as a cellular sensor of RNA virus infection resulting in typeI interferon induction. However, it is unknown antiviral response regarding interferon and regulation of inflammatory chemokines through RIG-I in oral keratinocytes and fibroblasts. Here, we examined the regulation of chemokines through RIG-I in immortalized oral keratinocytes (RT7) and fibroblasts (GT1) using real time PCR. RIG-I mRNA expressions were found in RT7 and GT1. The level of RIG mRNA in RT7 was increased by stimulating with IFN- $\gamma$. RIG-I ligands increased IFN- $\alpha, \beta$, CXCL10 and IL- 8 in both cells. Therefore, RIG-I in oral keratinocytes and fibroblasts may have important roles in the immune response in the regulation of typeI interferon and inflammatory chemokines.

\section{A-07. Correlations between the nutritional status of patients with cancer and the presence of candida} Hara K

Department of dentistry, Kitakyushu Municipal Medical Center

I examined the correlations between the nutritional status of inpatients with cancer and the presence of candida. Patients and Methods: I examined 491patients (304 males and 187 females). Their nutritional status was categorized as normal, slightly low, moderately low, and severely low, depending on their serum-albumin levels. The samples of oral microorganism were obtained from the entire oral mucosa and the saliva of the patients by sterile transport swabResultsAmong 133 patients with normal nutritional status, candida was detected in 42 patients (31.6\%). Among 358 patients with low nutritional status, candida was detected in 191 patients $(53.4 \%)$.
1A-08. Usefulness of Fungiflora $Y$ in rapid diagnosis of oral candidiasis

Okamoto $\mathrm{M}^{1}$, Kamoi $\mathrm{M}^{1}$, Yamachika $\mathrm{S}^{1,2}$, Yamamoto $\mathrm{K}^{1,3}$, Imamura $\mathrm{T}^{1,2}$, Kadomatsu $\mathrm{S}^{1,3}$, Saito $\mathrm{I}^{1,4}$, Maeda $\mathrm{N}^{1,5}$, Nakagawa $\mathrm{Y}^{1,2}$

${ }^{1}$ Dry Mouth Clinic, Tsurumi University Dental Hospital, ${ }^{2}$ Department of Oral and Maxillofacial Surgery, ${ }^{3}$ Department of Geriatric Dentistry, and ${ }^{4}$ Department of Pathology, ${ }^{5}$ Department of Oral microbiology, Tsurumi University School of Dental Medicine

Diagnosis of oral candidiasis is supported by the culture or the identification of spores and pseudohyphae in stained smears from the lesion. Fungiflora $\mathrm{Y}$ is a fluorescent stain, and has a specific affinity for chitin and cellulose, which are components of fungal cell wall.

Microscopic findings of Candida stained with Fungiflora $\mathrm{Y}$ were compared with CytoQuick stain. The results of present study suggested that Fungiflora $Y$ was useful chair side test for the rapid diagnosis of candidiasis.

1A-09. Antifungal drug susceptibility of oral Candida species isolated from our clinic

Yamazaki Y, Hata H, Murata T, Kitagawa Y

Oral Diagnosis and Medicine, Graduate School of Dental Medicine, Hokkido University

The prevalence of non-albicans candida species has been recently increasing as a cause of oral candidiasis and acquired azole resistance has been reported worldwide. To examine the difference of antifungal susceptibility between C.albicans and non-albicans candida species, we determined MICs in amphotericine B (AMPH-B), miconazole (MCZ) and itraconazole (ITCZ) against 82 strains of Candida species isolated from the oral cavities using commercially available antifungal susceptibility test.

C. albicans was susceptible to all three antifungal drugs, while non-albicans candida species, especially $C$.tropicalis was prone to acquire azole resistance.

1A-10. Genotype analysis of Candida albicans isolated from oral mucosa of healthy individuals Takagi Y, Fukano H, Kawahara K, Shimozato K Department of Maxillofacial Surgery, Aichi-Gakuin University School of Dentistry

Background: It is acknowledged that Candida albicans is a commensal yeast species in oral mucosa and causes superficial candidiasis in healthy individuals. Objective: In this study, we isolated multiple strains of $C$. albicans from a healthy people and analyzed their genotypes for study genotype variation in commensal site. Methods: $C$. albicans was isolated from oral mucosa of 71 healthy peoples and genotyped using 25S rDNA, RPS and microsatellite-based methods. Results: $C$. albicans was isolated from 31 peoples $(43.7 \%)$. Multiple genotypes were found in 17 peoples (55\%) who were positive to $C$. albicans. Conclusion: Genotype of $C$. albicans in commensal site is not homogenous. 
1A-11. Therapeutic effects of itraconazole oral solution on oral candidiasis

Oneyama $\mathrm{T}^{1}$, Tanaka $\mathrm{A}^{1}$, Toya $\mathrm{S}^{1}$, Mataga $\mathrm{I}^{2}$

${ }^{\prime}$ Department of Oral and Maxillofacial Surgery, Nippon Dental University Niigata Hospital

${ }^{2}$ Department of Oral and Mazillofacial Surgery, Nippon Dental University, School of Life Dentistry at Niigata

Oral solutions of the antifungal agent itraconazole were used in patients with oral disease being related to oral candidiasis. The subjects were 102 patients examined in our hospital for one year from April 2006 to May 2010. As a result, Candida was detected in 62 of the 102 patients $(60.8 \%)$. In the evaluation of results, the efficacy rates in the Candida detection group were $82.3 \%$ and $52.5 \%$ in the undetection group. Adverse events were found in 49 patients (52.9\%). Administration was discontinued in 11 patients $(10.8 \%)$. The itraconazole oral solution was effective against oral candidiasis.

1A-12. Occlusive therapy (OCT) with Miconazole gel (MCZ-gel), applied on denture mucosal surface is available for recurrent denture candidiasis (RDC) Kamikawa $\mathrm{Y}^{1}$, Nitta $\mathrm{T}^{1}$, Sakamoto $\mathrm{R}^{2}$, Nagayama $\mathrm{T}^{3}$, Mukai $\mathrm{H}^{2}$, Sugihara $\mathrm{K}^{2}$

${ }^{1}$ Department of Oral Surgery, Dental clinic, Kagoshima University Hospital.

${ }^{2}$ Department of Maxillofacial Diagnostic and Surgical Science, Kagoshima University Graduate School of Medical and Dental Science.

${ }^{3}$ Department of Applied Pharmacology, Kagoshima University Graduate School of Medical and Dental Science

Purpose: To clarify the availability of occlusive therapy (OCT) with Miconazol gel (MCZ-gel) for recurrent denture candidiasis (RDC). Subjects and Methods: Resin strips were immersed in Candida albicans and C.glabrata suspension liquid. Control group was applied vaseline and experimental group was applied MCZ-gel, and washed with Triton X100. Wash liquid were seeded on culture plate and measured $\mathrm{CFU} / \mathrm{ml}$. Eight cases of RDC were under went OCT with MCZ-gel. Results: CFU/ml of experimental group was significantly decreased compared with control group. All cases of RDC were not recognized more than 10 months. Conclusion: OCT with MCZ-gel may be available for RDC.

\section{A-13. Clinical investigation on oral lichen planus treated in our clinic}

Nakaya M, Ohno S, Morishita K, Sasabe E, Yamamoto T Department of Oral and Maxillofacial Surgery, Kochi Medical School, Kochi University

We investigated the clinical characteristics of 212 patients with oral lichen planus (OLP). Of the patients, 74\% were female and $26 \%$ were male. The mean age was 63.6 (range: 25-96) years. In total 314 lesions, the buccal mucosa was most predisposed, followed by the gingiva, tongue and lips. As the clinical type, reticular type is most predominant. Although topical steroid was applied to approximately $80 \%$ of the patients, patients with severe and refractory OLP were treated with oral administration or iontophoresis of steroids.
Seventy-six\% patients showed complete remission or improvement. Oral squamous cell carcinoma developed in 7 patients $(3.3 \%)$.

\section{A-14. An evaluation on classification of clinical} pattern of oral lichen planus

Hayashi $\mathrm{H}^{1}$, Jinbu $\mathrm{Y}^{1}$, Shinozaki $\mathrm{Y}^{2}$, Ito $\mathrm{H}^{1}$, Noguchi $\mathrm{T}^{1}$, Kusama $\mathbf{M}^{1}$

${ }^{1}$ Department of Dentistry, Oral and Maxillofacial Surgery, Jichi Medical University

${ }^{2}$ Department of Oral maxillofacial Surgery, Kyowa Central Hospital

Oral Lichen Planus(OLP) is diagnosed based on the clinical and histopathological criteria. Reproducible determination of clinical pattern is important for it's clinical investigation.

We studied correlation of clinical pattern determined by 5 special authorized dentists of Japan Society for Oral and Maxillofacial Surgery. Clinical photographs of 56 cases of OLP were categorized according to the classification by Axell, Siverman, Andreasen and Ito respectively, and the correlation of diagnosis among 5 clinicians was analyzed.

Good agreement was obtained in the cases of typical reticular pattern, however, there were striking differences in the other clinical patterns.

1A-15. Study on discrepancies between clinical and histopathological diagnosis in oral lichen planus

Nitta $T^{1}$, Kamikawa $Y^{1}$, Sakamoto $R^{1}$, Nagayama $T^{1}$, Mukai $\mathrm{H}^{1}$, Sugihara $\mathrm{K}^{1}$

${ }^{1}$ Department of Oral Surgery, Dental Clinic of Kagoshima University Medical and Dental hospital.

${ }^{2}$ Department of Applied Pharmacology, Kagoshima University Graduate School of Medical and Dental Sciences

The risk of oral cancer development for patient with oral lichen planus (OLP) is generally accepted. If the lesion is malignant tumor, early treatment is necessary. Discrepancies between clinical and histopathological diagnosis were investigated in 99 cases biopsied under the clinical diagnosis of OLP. The results revealed that rate of discrepancy was 20/99 (20.9\%). The most frequent histopathological diagnosis was squamous cell carcinoma and candidiasis. The former cases were most located at the lateral border of the tongue and the majority of these lesions were erosive type. These results indicated that biopsy is indispensable in clinically diagnosed OLP arising from these characters.

\section{A-16. Oral lichen planus: what are its histopatho-} logical criteria?

Saku $\mathrm{T}^{1,2}$, Maruyama $\mathrm{S}^{2}$, Yamazaki $\mathrm{M}^{1}$, Cheng $\mathrm{J}^{3}$

1Divisions of Oral Pathology, Niigata University Graduate School of Medical \& Dental Sciences, 2Oral Pathology Section, Department of Surgical Pathology, Niigata University Hospital

To make clear what were histopathologically diagnosed as oral lichen planus, we have surveyed cases of lichen planus as well as epithelial dysplasia in the surgical pathology file in our division during the last forty years from 1970 to 2009. Yearly proportions of cases diagnosed as 
lichen planus among the total cases have increased, as those of epithelial dysplasia did. This may be due to the introduction of immunohistochemistry to the diagnostic activity, which enabled us to distinguish epithelial dysplasia or carcinoma in-situ with lichenoid lymphocytic reactions from lichen planus. It is time to consider what lichen planus itself is as well as to establish a more reasonable disease entity of an oral mucositis type.

1A-17. Lack of perlecan deposits in the lymphocyte infiltrating zone of oral lichen planus: an immunohistochemical aid for its differential diagnosis from epithelial dysplasia

Maruyama $\mathrm{S}^{1}$, Yamazaki $\mathrm{M}^{2}$, Cheng $\mathrm{J}^{2}$, Saku $\mathrm{T}^{1,2}$

${ }^{1}$ Oral Pathology Section, Department of Surgical Pathology, Niigata University Hospital; ${ }^{2}$ Division of Oral Pathology, Niigata University Graduate School of Medical and Dental Sciences

Oral lichen planus (LP) is not always clearly diagnosed on hematoxylin and eosin (HE)-stained tissue sections, because similar histopathological features are shared by epithelial dysplasia (ED) with lichenoid reactions. To develop a diagnostic aid for LP, we compared immunohistochemical profiles in 16 clinically LP-diagnosed cases that presented a diagnostic challenge. Among them, 10 cases were finally diaganosed as LP and 6 as ED. There were no definite differences in lymphocytic subset populations, though perlecan was not positive in the characteristic bandlike zone of lymphocytic infiltration in LP. In contrast, there was no such clear perlecan-lacking zone in ED with lichenoid reactions. It is therefore possible to make a differential diagnosis of LP and ED by perlecan immunohistochemistry.

\section{A-18. Expression pattern of keratin 17 in oral lichen planus}

Yamazaki $\mathbf{M}^{1}$, Maruyama $\mathrm{S}^{2}$, Cheng $\mathrm{J}^{1}$, Saku $\mathrm{T}^{1,2}$

${ }^{1}$ Division of Oral Pathology, Niigata University Graduate School of Medical and Dental Sciences; ${ }^{2}$ Department of Surgical Pathology, Oral Pathology Section, Niigata University Medical and Dental Hospital

Oral lichen planus (LP) is not always clearly diagnosed on hematoxylin and eosin (HE)-stained tissue sections, because similar histopathological features are shared by epithelial dysplasia with lichenoid reactions (LD). To develop a diagnostic aid for LP, we compared immunohistochemical profiles for keratin17 (K17) in 16 clinically LP-diagnosed cases that presented a diagnostic challenge. Although K17 was expressed in the epithelium in all of the LP and LD cases examined, K17-positive cell debris scattered in the subepithelial connective tissue zone were more frequently observed in $\operatorname{LP}(9 / 11,91 \%)$ than in $\operatorname{LD}(2 / 6$, $33 \%$ ) with statistical significance. These results suggested that the identification of K17-positive cell fragments, that may correspond to so-called Civatte bodies, is useful for the differential diagnosis of LP from LD.
1A-19. Role of PGE2 and dendritic cells in oral lichen planus

Mukae $\mathrm{S}^{1}$, Nagai $\mathrm{K}^{1}$, Omagari $\mathrm{D}^{1}$, Matsumoto $\mathrm{N}^{1}$, Asano $\mathrm{M}^{1}$, Oki $\mathrm{H}^{2}$, Komiyama $\mathrm{K}^{1}$

Departments of ${ }^{1}$ Pathology, ${ }^{2}$ Oral Maxillofacial Surgery, Nihon University School of Dentistry

To clarified disease state of oral lichen planus, we performed an immunohistochemistry, RT-PCR, proteomics analysis of the lesion and have developed mouse models. The data suggested that CD4+ and CD8+ cells were identified as a major player in the cell infiltration. Particular amounts of NK cells and mature dendritic cells appeared as a regulator for immune response. The covering epithelium revealed secretory phosphlipase A2 and COX-2, which indicates PGE2 synthesis from the disease epithelial cells. These PGE2 may involved dendritic cell maturation that was confirmed by mouse DC models. The result indicated that PGE2 synthesis from disease epithelium play an important role to develop and maintain the lesion through the maturation of dendritic cells.

\section{A-20. Treatment of erosive/ulcerative oral lichen planus with topical tacrolimus}

Takahashi $\mathrm{G}^{1}$, Akagaki $\mathrm{S}^{2}$, Iwai $\mathrm{S}^{1}$, Yura $\mathrm{Y}^{1}$

${ }^{1}$ Department of Oral and Maxillofacial Surgery II, Osaka University Graduate School of Dentistry.

${ }^{2}$ Department of Oral and Maxillofacial Surgery, Toyonaka Municipal Hospital

Oral lichen planus (OLP) is a chronic inflammatory disease of the oral mucosa. Erosive/ulcerative type of OLP is often painful and is refractory to available topical corticosteroid therapy. We reported 20 patients who had erosive/ ulcerative OLP and were treated with $0.1 \%$ tacrolimus ointment in this Department during the period from 2003 to 2009. All the patients had buccal mucosa lesions and other lesion were observed in the gingiva, tongue, lower lip and palate. When the effectiveness of topical tacrolimus was classified dependent on the degree of clinical improvement, the percentage of good response was $80 \%(16 / 20)$. Three patients stopped to use the drug because of burning sensation and local irritation, but no serious adverse side effect was observed. In 12 cases, relapse occurred within the average period of 8.8 weeks. Topical tacrolimus was effective for the treatment of erosive/ulcerative OLP refractory to local corticosteroid therapy.

1A-21. A case of squamous cell carcinoma in the dorsum of tongue after seven months application of tacrolimus for oral lichen planus

Sato $\mathrm{T}^{1}$, Yamazaki $\mathrm{Y}^{1}$, Murata $\mathrm{T}^{1}$, Abe $\mathrm{T}^{1}$, Kitamura $\mathrm{T}^{2}$, Shindoh $\mathrm{M}^{2}$, Kitagawa $\mathrm{Y}^{1}$

${ }^{1}$ Oral Diagnosis and Medicine, Department of Oral Pathobiological Science, Hokkaido University Graduate School of Dental Medicine.

${ }^{2}$ Department of Oral Pathology and Biology, Hokkaido University Graduate School of Dental Medicine

Oral lichen planus (OLP) is a mucocutaneous chronic inflammatory condition. However, some OLP lesions are 
classified into a precancerous condition, etiology remains unclear.

An 80 years-old-woman presented with erosive and ulcerative lesions on bilateral buccal regions and a part of dorsum of tongue. A biopsy showed OLP. An application of topical steroid was ineffective, and the tongue lesion was progressing to the whole dorsum of tongue. Then, topical tacrolimus ointment was applied. Although the buccal lesions were decreased in size, the lesion on dorsum of tongue was accompanied by induration 7 months after the use of tacrolimus ointment. The second biopy from the tongue proved to be squamous cell carcinoma.

\section{A-22. A case of bullous lichen planus assumed oral lesion first symptoms}

Murata T, Yamazaki Y, Sato T, Goto J, Kitagawa Y

Oral Diagnosis and Oral Medicine, Department of Oral Pathobiological Science, Graduate School of Dental Medicine, Hokkaido University

Bullous lichen planus is a rare autoimmune blistering disease. We report a case of oral bullous lichen planus in a 73-years-old woman. Oral manifestation might be the first symptom in this case. Biopsy was performed from oral and skin bullous lesions. Histopathologically, these lesions were diagnosed lichen planus. When we consulted with Dermatology, hepatitis C, drug and metal allergy were considered as a cause of the bullous lichen planus. By solving each problem, the symptoms are improving.

\section{A-01. A case of osteoplastic epulis in a 4-year-old child}

Furuya $\mathrm{I}^{1}$, Shibasaki $\mathrm{M}^{1}$, Hoshi $\mathrm{H}^{1}$, Takeda $\mathrm{Y}^{2}$, Sugiyama $\mathrm{Y}^{1}$

${ }^{I}$ Division of Oral Surgery, Department of Oral and Maxillofacial Surgery, School of Dentistry, Iwate Medical University.

${ }^{2}$ Division of Oral Pathology, Department of Oral Etiology and Pathological Regulation, School of Dentistry, Iwate Medical University

A 4-year-old girl had a swelling of her gingiva in maxillary primary incisor lesion for one year. She visited our hospital in March, 2008, because the swelling had not disappeared. As we considered the swelling might be caused by periapical periodontitis, and we performed root canal treatment of the left maxillary primary incisor. The symptom, however, did not disappear. Thus the patient was given a diagnosis of tumor or epulis, and surgical excision of the lesion was performed. The pathological diagnosis of osteoplastic epulis was made. There is no sign of recurrence 8 months postoperatively.

2A-02. Two cases of pyogenic granuloma in a child and a clinico-statistical study of pyogenic granuloma in our department

Yamashita M, Jinbu Y, Ioka Y, Miyagi N, Itoh H, Kusama M

Department of Dentistry, Oral and Maxillofacial Surgery, Jichi Medical University

Pyogenic granuloma is a non-neoplastic inflammatory hyperplasia of the skin and mucosa that is relatively common in the oral cavity, but rare in children. The exact pathogenesis of this condition remains obscure and a differential diagnosis is often complicated by rapid growth.

We describe a 2-year-old boy (Case 1) with an exophytic pedunculated small mass on the right margin of the tongue and a 12-year-old boy (Case 2) with a large exophytic mass on the mandible. Both lesions were surgically resected and histopathologically diagnosed as pyogenic granuloma.

A clinico-statistical study of this condition within our department is also presented.

\section{A-03. A case of nasal polyp suspected neoplastic} lesion

Kamitani M, Tsuji K, Iwamoto S, Shima M

Oral Surgery, Kishiwada City Hospital

Nasal polyp is a disease that accompanied as one case of chronic sinusitis, aspirin asthma and allergic rhinitis. The patient was 18-years-old woman with nasal congestion and diffuse swelling of left cheek. Radiograph showed root absorption of upper jaw first molar. CT and MRI findings showed mass which suffused left side maxillary sinus and expanded to right side nasal cavity. First impression of those clinical findings was odontogenic tumor, but the pathological diagnosis by biopsy was nasal polyp. Surgical operation was done, the lesion was solid and had adhered to nasal cavity mucosa partly. After the operation, nasal congestion improved.

2A-04. A case of linear scleroderma of the lip accompany with alveolar bone resorption

Saisu $\mathrm{H}^{1}$, Tsunoda $\mathrm{K}^{1}$, Fukaya $\mathrm{C}^{1}$, Yamada $\mathrm{Y}^{1}$, Adachi $\mathrm{T}^{2}$, Amagai $\mathrm{M}^{2}$, Nakagawa $\mathrm{T}^{1}$

${ }^{1}$ Departments of Dentistry and Oral Surgery and ${ }^{2}$ Dermatology, Keio University School of Medicine

Linear scleroderma (LS) is localized skin sclerosis with dyschromatosis. Development of LS lesion on lip is rare. A 12-year-old female noticed linear whitish lesion on middle of lower lip without clear diagnosis. Recession of lower central incisor gingiva with deep periodontal pocket formation was also developed. Thus the patient referred to our clinic for evaluation. Skin biopsy revealed swelling and homogenization of collagenous fiber. From these clinical and pathological results, we diagnosed this patient as LS. It is necessary to confirm oral mucosal symptoms when encounter the patient with skin lesion around the mouth.

\section{A-05. A case report of Paracoccidioidomycosis patient required differential diagnosis of oral malignant tumor}

Fujihara $\mathrm{H}^{1}$, Yamada $\mathrm{H}^{1}$, Terada $\mathrm{C}^{2}$, Kamiya $\mathrm{Y}^{2}$, Tanaka $\mathrm{Y}^{1}$, Asada $\mathrm{K}^{2}$, Satomura $\mathrm{K}^{2}$, Hamada $\mathrm{Y}^{1}$

${ }^{1}$ Department of Oral and Maxillofacial Surgery, School of Dental Medicine, Tsurumi University. ${ }^{2}$ Department of Oral Medicine and Stomatology, School of Dental Medicine, Tsurumi University

Paracoccidioidomycosis is one of fungus infection frequently reported from Southern American Countries but 
quite rare in Japan. However, with the increasing number of passengers to abroad and immigrations from those areas, patients of so-called imported infectious diseases have been increased.

In this report, we describe a case of a 52-old-man with Paracoccidioides brasiliensis developed in the oral mucosa. He had suffered from pain of lower lip and trismus. His general condition was quite poor and lesions of lower lip resembled to ulcerative carcinoma. This case would suggest us the knowledge of differential diagnosis of carcinoma and rare imported infectious diseases.

\section{A-06. Comprehensive analysis of keratin expres- sion in oral squamous cell carcinoma and epithelial dysplasia}

Sakamoto $\mathrm{K}^{1}$, Morita $\mathrm{K}^{2}$, Omura $\mathrm{K}^{2}$, Yamaguchi A ${ }^{1}$

${ }^{1}$ Section of Oral Pathology, Graduate School of Tokyo Medical and Dental University

${ }^{2}$ Section of Oral and Maxillofacial Surgery

Aim) This study aimed to elucidate the significance of alteration of keratin expression in the pathogenesis of oral squamous cell carcinoma (OSCC) and epithelial dysplasia (OED). Methods) cDNA microarray analyses on all the keratins were performed using microdissected samples of 43 OSCC cases. Immunohistochemical examination of major epithelial keratins was performed on 10 cases of OSCC associated with OED. Results) Keratin 4,13, 15 and 19 were downregulated, whereas keratin 5, 6, 14, 16 and 17 were upregulated in OSCC and OED. The significance of these systematic alterations and application of the keratin profiling to pathological diagnosis will be discussed.

\section{A-07. Dkk-3 protein expression in head and neck squamous cell carcinoma}

Katase N, Lefeuvre M, Sathi GA, Hirata Y, Matsuda H, Kubota M, Nagatsuka $\mathrm{H}$

Department of Oral pathology and medicine, Graduate School of Medicine, Dentistry and Pharmaceutical Sciences, Okayama University

We previously reported that frequent loss of heterozygosity (LOH) was detected in Dkk-3 locus in Head and neck squamous cell carcinoma (HNSCC). In the present study, we investigated Dkk-3 protein expression in HNSCC tissue samples and cell lines. Immunohistochemistry revealed that 76/ 90 cases $(84.4 \%$ ) of the HNSCC expressed Dkk-3 protein. Moreover, all the HNSCC derived cell lines expressed Dkk-3 protein in various degrees. Interestingly, Dkk-3 (-) showed significantly longer disease free survivals $(\mathrm{p}=0.038)$. Taken together, Dkk-3 is thought to play an important role in cancer progression of HNSCC, and Dkk-3 protein expression might be a prognostic factor.
2A-08. Relationships of cervical lymph node metastasis to lymphatic invasion in tongue squamous cell carcinoma

Okada $\mathrm{Y}^{1,2}$, Kanri $\mathrm{Y}^{1}$, Ohkubo $\mathrm{Y}^{1}$

${ }^{1}$ Department of Pathology, The Nippon Dental University, School of Life Dentistry at Niigata

${ }^{2}$ Clinical Laboratory, The Nippon Dental University Niigata Hospital

This study aimed to identify predictive factors for cervical lymph node (CLN) metastasis in tongue squamous cell carcinoma (SCC). Initial biopsy specimens obtained from 30 patients with tongue SCC were examined to evaluate histological malignancy according to Anneroth's classification. In addition, lymph vessel density, and lymphatic invasion in tumor were evaluated immunohistochemically using the D2-40 and AE1/AE3, and then the relationships of CLN metastasis to these parameters were investigated. Histological malignancy grade and lymphatic invasion were related significantly with CLN metastasis $(\mathrm{p}<0.05)$, but there was no relation between lymph vessel density and CLN metastasis.

\section{A-09. Current state of oral cancer detecting system ICHIKAWA network (OCDSIN)}

Tanaka $\mathrm{Y}^{1}$, Sato $\mathrm{K}^{2}$, Kamiyama $\mathrm{I}^{2}$, Katakura $\mathrm{A}^{2,3}$, Yamashina $\mathrm{M}^{4}$, Tonogi $\mathrm{M}^{4}$, Yamane $\mathrm{G}^{2,4}$

${ }^{1}$ Division of Clinical Laboratory, Ichikawa General Hospital, Tokyo Dental College,

${ }^{2}$ Tokyo Dental College Oral Cancer Center, ${ }^{3}$ Division of Oral and Maxillofacial Surgery, Department of Clinical Oral Health Science, Tokyo Dental College, ${ }^{4}$ Department of Oral Medicine, Oral and Maxillofacial Surgery, Tokyo Dental College

We considered that examination of the oral mucosa during dental treatment in a dental clinic is one of the most efficient methods of oral cancer detecting system. Based on this, in Ichikawa city, we started a screening system in a dental clinic run by members of the Ichikawa Dental Association in 2007. In this system, liquid-based cytology was undertaken by the dentists. The maneuvers needed to obtain specimens for the liquid-based cytology provided the opportunity to the dentists for examination of the oral mucosa, and was useful to obtain good-quality specimens regardless of the source of the specimens.

\section{A-10. A study of 38 oral leukoplakias with malig- nant transformation}

Amagasa T, Yamashiro M, Uzawa N, Michi Y, Igarashi $\mathrm{H}$, Suzuki T

Maxillofacial Surgery, Graduate School, Tokyo Medical and Dental University

We will report 38 oral leukoplakias with malignant transformation. Twenty one out of 38 patients were female and 17 were male. The age of the patients who were diagnosed as carcinoma ranged from 27 to 83 years. As for the clinical type of leukoplakia at first visit, 20 were classified to mixed type (type II) and 14 were white patch (Type I). As for the treatment of leukoplakia, 14 leukoplakias were treated by nothing, 9 by chemotherapy, 5 by radiation. 
As for the site of cancer, 26 were found on the tongue, 6 on the buccal mucosa and 5 on the lower gum. The periods from oral leukoplakia to malignant transformation ranged from 5 months to 25 years.

\section{A-11. Intraductal papilloma with carcinomatous} foci of the palate: a case report

Funayama $\mathrm{A}^{1}$, Maruyama $\mathrm{S}^{2}$, Cheng $\mathrm{J}^{3}$, Yoshizawa $\mathrm{M}^{1}$, Oda $\mathrm{Y}^{1}$, Shingaki $\mathrm{S}^{1}$, Saito $\mathrm{C}^{1}$, Nishiyama $\mathrm{H}^{4}$, Hayashi $\mathrm{T}^{4}$, Saku $\mathrm{T}^{2,3}$

Divisions of ${ }^{1}$ Reconstructive Surgery for Oral and Maxillofacial Region, ${ }^{2}$ Oral and Maxillofacial Radiology, and ${ }^{3}$ Oral Pathology, Niigata University Graduate School of Medical and Dental Sciences; ${ }^{4}$ Oral Pathology Section, Department of Surgical Pathology, Niigata University Hospital

We report a case of intraductal papilloma with carcinomatous foci. A 73-year-old female had noticed a painless swelling, measuring $3 \times 9 \times 8 \mathrm{~mm}$ in size, in her palate for seven months. The tumor was surgically removed. Histopathologically, the tumor was composed of two parts of different cell type compositions. In the mucosal surface part, the tumor consisted of papillary proliferation of ductal cells in a two-cell layer arrangement with keratin 19+ inner and calponin+ basal ones borne on fibrovascular cores within a dilated ductal space. In the deeper portion, however, there were only solid cord-like or small round-shaped tumor cell nests invading surrounding mucous gland tissues. The deeper part was considered to be a secondarily arising carcinomatous focus in intraductal papilloma.

\section{A-12. A case of acute leukemia diagnosed in an infant with gingival swelling \\ Fukuchi K, Oishi K, Kuroda T \\ Department of Oral and Maxillofacial Surgery, Osaka City General Hospital}

We report a case of acute leukemia an infant who presented with gingival swelling. A 10-month-old girl visited our hospital due to swelling of the lower gingiva. An intraoral examination revealed swelling of the gingiva in the lower left jaw region. A blood examination showed anemia and thrombocytopenia and so a systematic leukemia (AML:M5) was made following a bone marrow examination. The patient underwent chemotherapy and the swelling of the gingival completely disappeared after the first treatment.

\section{A-13. Report of a case with oropharyngeal cancer complained of neck mass}

Sato $\mathrm{Y}^{1}$, Yamamoto $\mathrm{N}^{1}$, Kimura $\mathrm{N}^{1}$, Sasaki $\mathrm{T}^{2}$, Fukushima $\mathrm{H}^{2}$, Yonekawa $\mathrm{H}^{2}$, Mitani $\mathrm{H}^{2}$, Kawabata $\mathrm{K}^{2}$, Ishikawa $\mathrm{Y}^{1}$

${ }^{I}$ Division of Pathology, Cancer Institute of Japanese Foundation for Cancer Research, Japan

${ }^{2}$ Devision of Head and Neck, Cancer Institute Hospital, Japan

The patient is a 47-years-old male who presented with a left-sided cervical nodes containing metastatic SCC. Clinical examination and CT/MRI/PET revealed no obvious primary mucosal lesion. He went on to have bilateral tonsillectomies, a left modified radical neck dissection. Left tonsil specimen contained small foci of SCC. Cancer of unknown primary site with an incidence of $1-5 \%$ is a separate entity in the classification of head and neck tumors. It is well known that the tonsil may be the site of an occult primary tumor. Tonsillar carcinomas have a tendency to form cystic metastases. This knowledge can greatly assist dentists in identifying lesions that may require treatment in the general hospital.

2A-14. A case report of an extrapleural solitary fibrous tumor arising from the floor of mouth

Nobusawa $A^{1}$, Yamaguchi $\mathrm{T}^{1}$, Negishi $\mathrm{A}^{1}$, Yokoo $\mathrm{S}^{1}$, Sano $\mathrm{T}^{2}$, Hirato $\mathrm{J}^{3}$, Oyama $\mathrm{T}^{2}$

${ }^{1}$ Department of Stomatology and Oral Surgery Gunma University Graduate School of Medicine.

${ }^{2}$ Department of Diagnostic Pathology Gunma University Graduate School of Medicine.

${ }^{3}$ Department of Pathology Gunma University Hospital

Solitary fibrous tumor (SFT) is an uncommon benign neoplasm reported as a tumor of the pleura. But now, it has been found that SFT can be occurred in other site of the body, such as digestive tract, parenchymal organ, intracranial cavity and soft tissue. They are called extrapleural solitary fibrous tumors. We report a case of an extrapleural SFT arising from the floor of mouth in our Hospital. Histopathological examination of the surgical specimen revealed encapsulated fibrous mass with septum. Immunohistopathologically, tumor was consisted with 2 regions -benign and malignant- separated by the septum.

\section{A-15. Symmetric lipomatosis of the tongue : report of a case}

Kato R, Jinbu Y, Ioka Y, Miyagi N, Kusama M

Department of Dentistry, Oral and Maxillofacial Surgery, Jichi Medical University

Lipoma is one of the non-epithelial benign tumors occasionally seen in the oral cavity. However, symmetric lipomatosis is an extremely rare disease in the oral cavity.

A case of symmetric lipomatosis of the tongue is reported. The patient was a 73-year-old male who complained of a bilateral swelling of the tongue. The tongue of the patient was diffusely and markedly enlarged with somewhat nodular appearance in the lateral borders, presenting as a macroglossia. Adipose tissues were interspersed with the muscles fibers and distinct capsule formation was not found clinically and histopathologically, the patient was finally diagnosed as having symmetric lipomatosis of the tongue.

\section{A-16. A case of angioleiomyoma on the palate} Takagi $\mathrm{S}^{1}$, Ohmi $\mathrm{Y}^{1}$, Kubota $\mathrm{N}^{2}$, Kubota $\mathrm{E}^{1}$

${ }^{1}$ Department of Maxillofacial Surgery, Kanagawa Dental College

${ }^{2}$ Department of Diagnostic Science Division of Pathology, Kanagawa Dental College

Angioleiomyoma is benign tumor derived from the smooth muscle and common in lower limbs, uterus and gastrointestinal tract. However, it rarely occurs in the oral area because few of smooth muscle observed in oral tissue. 
We present a case of angioleiomyoma, which occurred as a lump on the palate. Case: Seventy three-year-old woman came to our department complaining the lump on the palate. She has noticed it from two years ago. The lump was admitted as a hemisphere of cob $(15 \times 15 \mathrm{~mm})$. The tumor was surgically excised under local anesthesia. Histopathological findings revealed that a large number of vascular cavities existed in smooth muscle. Smooth muscle proliferation was markedly observed around the vascular tissues. Angioleiomyoma was thus diagnosed. Currently, the patient is sound and no recurrence of the tumor was observed.

\section{A-17. A case of ameloblastoma granular cell type} Hara $\mathrm{A}^{1}$, Yamaguchi $\mathrm{A}^{1}$, Kokubun $\mathrm{K}^{1}$, Enokiya $\mathrm{Y}^{1}$, Muramatsu $\mathrm{T}^{1}$, Hashimoto $\mathrm{S}^{1}$, Matsuzaka $\mathrm{K}^{2}$, Shibahara ${ }^{3}$, Inoue $\mathrm{T}^{2}$, Shimono $\mathrm{M}^{1}$

${ }^{I}$ Department of Pathology, Tokyo Dental College

${ }^{2}$ Department of Clinical Pathophysiology, Tokyo Dental College ${ }^{3}$ Department of Oral \& Maxillofacial Surgery, Tokyo Dental College

Ameloblastoma granular cell type is a rare tumor which accounted for $5 \%$ of all ameloblastomas. It tends to occur in elders in comparison with usual ameloblastomas. We present a case of ameloblastoma granular cell type in the right mandible of an 80-years-old man. Radiographic examination showed a unicystic radiolucent lesion. Histopathologically, lining epithelium was composed of cells with acidophilic granules. Immunohistochemically, the granular cells were positive for AE1/AE3, CK13, and CK19, and histochemically, its cytoplasm was positive for PAS. Its characteristics are discussed in term of its histochemical and immunohistochemical features.

2A-18. Comprehensive keratin profiling reveals different histopathogenesis of keratocystic odontogenic tumor and orthokeratinized odontogenic cyst Aragaki $\mathrm{T}^{1}$, Sakamoto $\mathrm{K}^{2}$, Katsube $\mathrm{K}^{2}$, Amagasa $\mathrm{T}^{1}$, Yamaguchi $\mathrm{A}^{2}$

${ }^{1}$ Section of Maxillofacial Surgery, Graduate School of Tokyo Medical and Dental University. ${ }^{2}$ Section of Oral Pathology of Tokyo Medical and Dental University

To better understand the pathogenesis of keratocystic odontogenic tumor (KCOT) and orthokeratinized odontogenic cyst (OOC), comprehensive profiling of keratin expression was performed. OOC expressed keratin 1 (K1), $\mathrm{K} 2$, K10 and loricrin, suggesting differentiation toward normal epidermis. KCOT expressed K4, K13, K17 and K19, which is reminiscent of a mucosal squamous epithelium and an epithelial appendage. Cells strongly positive for K17 and K19 were also observed specifically in the dental lamina cells, implying the origin of KCOT. GLI2 was significantly expressed in KCOT, accompanied with robust K17, mTOR and BCL2 expression. These findings suggest their characteristic histology, pathogenesis and biological behaviors.
2A-19. Comparison of orthokeratinized odontogenic cyst and facial epidermis in cytokeratine, Bcl-2 and Bax

Hayashi $\mathrm{T}^{1}$, Wato $\mathrm{M}^{2}$, Tsuji $\mathrm{K}^{1}$, Iseki $\mathrm{T}^{1}$, Tanaka $\mathrm{A}^{2}$, Morita $\mathrm{S}^{1}$

${ }^{1}$ First Department of Oral and Maxillofacial Surgery, Osaka Dental University

${ }^{2}$ Department of Oral Pathology, Osaka Dental University

Lining epithelium of odontogenic keratocysts are divided into parakeratosis and orthokeratosis. WHO classified odontogenic keratocyst with parakeratosis as keratocystic odontogenic tumor (KCOT) in 2005. And odontogenic keratocyst with orthokeratosis was not classified as odontogenic tumor, though it is also referred to as orthokeratinized odontogenic cyst (OOC). We investigated the biological characteristics of KCOT, OOC and facial epidermis. This was conducted by immunohistochemical studies with CK, Bcl-2 and Bax. There were deferences in the expression of $\mathrm{CK}, \mathrm{Bcl}-2$ and Bax between the lining epithelium of OOC and KCOT.

\section{A-20. A case of bilateral salivary glands swelling masses}

Sato $\mathrm{S}^{1}$, Kishino $\mathrm{M}^{1}$, Ogawa $\mathrm{Y}^{1}$, Ogawa $\mathrm{I}^{2}$, Takata $\mathrm{T}^{3}$, Toyosawa $\mathrm{S}^{1}$

${ }^{1}$ Department of Oral Pathology, Graduate School of Dentistry, Osaka University, ${ }^{2}$ Center of Oral Clinical Examination, Hiroshima University Hospital, ${ }^{3}$ Division of Frontier Medical Science, Department of Oral and Maxillofacial Pathobiology, Graduate School of Biomedical Sciences, Hiroshima University

An 80-year-old woman was admitted because of painless swellings in bilateral parotid regions. Histologically, the lesional nodules had incomplete capsules and engulfed the surrounding parotid glands at the periphery. The lesions were composed of clear cells and oncocytic cells with abundant fine granules. This case has multiple nodules in bilateral glands, however, no evidence of malignant histological findings was found. The clear cells, as well as the oncocytic cells, were positive to PAS and PTAH staining. The cells were diffusely reactive with anti-mitochondrial antibody and cytokeratin AE1/AE3.

\section{B-07. A case of contact cheilitis due to cosmetics Yoshizawa $\mathrm{M}$}

Division of Reconstructive Surgery for Oral and Maxillofacial Region, Department of Tissue Regeneration and Reconstruction, Course for Oral Life Science, Niigata University Graduate School of Medical and Dental Sciences

Lipsticks, toothpaste, and foods are common causes of contact cheilitis. We report a case of contact cheilitis probably due to cosmetics. A 37-year-old female had suffered from itching and reddish swelling of her lips since Jun 2009 and consulted our department on October. After the first visit, small blisters were prominent on lips and erythema and papules appeared on neck and forearm. She showed positive reactions to foundation which she had used since June and diisostearyl malate which is one of the constituents of lipsticks in patch testing. She stopped using these 
cosmetics then, her cheilitis improved within one week.

\section{B-08. A case of Stevens-Johnson syndrome thought to be caused by allopurinol}

Fujimoto $\mathrm{M}^{1}$, Ukichi $\mathrm{K}^{1}$, Takada $\mathrm{A}^{1}$, Fukushima $\mathrm{T}^{1}$, Morimoto $\mathrm{M}^{1}$, Yamane $\mathrm{G}^{1}$, Tanaka $\mathrm{Y}^{2}$, Yosida $\mathrm{R}^{3}$, Takahashi $\mathrm{S}^{3}$

Department of Oral Medicine, Oral and Maxillofacial Surgery, Tokyo Dental College ${ }^{l}$

Department of Clinical Laboratory,Division of Surgical Pathology, Ichikawa General Hospital, Tokyo Dental College ${ }^{2}$ Department of Dermatology, Ichikawa General Hospital, Tokyo Dental College ${ }^{3}$

We report a case of Stevens-Johnson syndrome thought to be caused by allopurinol. The patient was a 70-year-old man who complained of hemorrhagic bullae and erosions of whole body accompanied by bloody crusts in the eyes and oral cavity. We cleaned the mouth with dentist and hygienist. We observed oral symptomatic improvement from mouth cleaning start the eight day. Mucocutaneous symptoms improved only with transfusion, ointment application on the skin and eyes and mucocutaneous care until the $18^{\text {th }}$ day after admission. Because he accompanied diabetes mellitus with poor control and pneumonia, We did not use steroid in this case.

1B-09. A case of neonatal oral mucosal ulcer suspected of food protein-induced enterocolitis of infancy

Ihara $\mathrm{Y}^{1}$, Tsunoda $\mathrm{K}^{1}$, Kato $\mathrm{S}^{1}$, Hyodo $\mathrm{T}^{1}$, Nakagawa $\mathrm{T}^{1}$, Morita $\mathrm{H}^{2,3}$, Nagai $\mathrm{T}^{1}$

Departments of ${ }^{1}$ Dentistry and Oral Surgery and ${ }^{2}$ Pediatrics, Keio University School of Medicine. ${ }^{3}$ National Research Institute for Child Health and Development, Department of Allergy and Immunology

Food protein-induced enterocolitis of infancy (FPIES) is characterized as vomit and bloody stool just after starting of lactation. A neonatal female was hospitalized to pediatrics by vomit and pyrexia after lactation. These symptoms improved by stopping of lactation, however pyrexia recurred and ulcers on tongue and soft palate were observed when restarting of lactation. From these progresses, we suspected her as milk allergy. Lactation of hydrolyzed milk leads reduction of symptoms. Antigen specific DLST test revealed positive against casein. These facts suggest that oral ulcer was caused by FPIES. FPIES is one of candidate for diagnosing of neonatal oral ulcer.

1B-10. A case of oral mucous membrane ulcers and ageusia caused by disease-modifying antirheumatic drug (DMARD; bucillamine)

Sumida $E^{1}$, Araseki A ${ }^{2}$, Takahashi E ${ }^{2}$

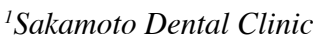

${ }^{2}$ Department of Dentistry and Oral Surgery, Kawasaki Social Insurance Hospital

We report a patient with oral mucous membrane ulcers and ageusia induced by disease modifying antirheumatic drug (DMARD; bucillamine)
A 65-year-old woman was diagnosed as rheumatoid arthritis (RA) and had been taking bucillamine for 11 months. Oral mucous membrane ulcers were observed on the tongue. After bucillamine was discontinued, the oral mucosa improved in 47days.

\section{B-11. A case of mucous membrane plasmacytosis} of the gingival

Ueno $\mathrm{S}^{1}$, Ogawa $\mathrm{Y}^{1}$, Fukuda $\mathrm{A}^{1}$, Wato $\mathrm{M}^{2}$, Morita $\mathrm{S}^{1}$

${ }^{\prime}$ First Department of Oral and Maxillofacial Surgery, Osaka Dental University

${ }^{2}$ Department of Oral Pathology, Osaka Dental University

Mucous membrane plasmacytosis is an unusual nonneoplastic condition involving plasma cell proliferation. The precise etiology, pathogenesis, and treatment of the lesions are unknown. A case of mucous membrane plasmacytosis in the upper and lower gingiva of a 35-year-old Japanese woman is presented. The patient visited our clinic complaining of a reddish swelling in the upper and lower gingiva. Histopathologically, the lesion was diognosed mucous membrane plasmacytosis because of dense infiltration of plasma cells without cellular atypia in the submucosa. Topically appliance of a steroid ointment to the lesion was effective.

\section{B-12. Plasmocytosis circumorificialis successfully} treated by sodium fusidate

\section{Ueno Y}

Department of Dentistry, Oral and Maxillofacial Surgery, Jichi Medical University

Plasmocytosis circumorificialis (PC) is a rare inflammatory mucosal condition histologically characterized by a dense plasma cell infiltration in the mucosa. The patient was a 74-year-old man who presented with symptoms on his lower lip of erythematous erosion with crust formation and diffuses edematous swelling. A biopsy specimen showed dense inflammatory cell infiltration mainly comprising plasma cells. Immunohistochemically, these cells were positive for immunoglobulin (Ig) A, strongly positive for $\mathrm{IgG}$, positive for IgM, and strongly positive for kappa and lambda chains. According to histopathological and clinical findings, the lesion was diagnosed as PC. The patient was treated using a sodium fusidate, and symptoms gradually improved and then resolved completely. 


\section{B-01. Clinical examinations about the setting of the diagnostic criterion value of dry mouth using an oral moisture checking device}

Fukushima $\mathrm{Y}^{1}$, Yoda $\mathrm{T}^{1}$, Araki $^{2}$, Murata $^{3}$, Kitagawa $^{3}$, Omura $\mathrm{K}^{4}$, Toya $\mathrm{S}^{5}$, Ito $\mathrm{K}^{6}$, Funayama $\mathrm{S}^{6}$, Iwabuchi $\mathrm{H}^{7}$, Asano $\mathrm{K}^{8}$, Imai $\mathrm{Y}^{8}$, Negishi $\mathrm{A}^{9}$, Yokoo $\mathrm{S}^{9}$, Matsumoto $\mathrm{G}^{10}$, Kubota $\mathrm{E}^{10}$, Watanabe $\mathrm{H}^{11}$, Kusama $\mathrm{M}^{11}$, Onizawa $\mathrm{K}^{12}$, Goto $\mathrm{T}^{12}$, Shinozaki $\mathrm{S}^{13}$, Nakamura $\mathrm{S}^{13}$, Nakazawa $\mathrm{R}^{14}$, Harada $\mathrm{K}^{14}$, Fujibayashi $\mathrm{T}^{10}$

${ }^{1}$ Department of Oral and Maxillofacial Surgery, Faculty of Medicine, Saitama Medical University, ${ }^{2}$ Community Health Science Center, Saitama Medical University, ${ }^{3}$ Oral Diagnosis and Oral Medicine, Department of Oral Pathobiological Science, Graduate School of Dental Medicine, Hokkaido University, ${ }^{4}$ Oral and Maxillofacial Surgery, Department of Oral Restitution,Division of Oral Health Sciences, Graduate School, Tokyo Medical and Dental University, ${ }^{5}$ Oral and Maxillofacial Surgery, Dry Mouth Clinic, The Nippon Dental University Niigata Hospital, ${ }^{6}$ Division of Gereatric Dentistry, Niigata University Medical \& Dental Hospital, ${ }^{7}$ Department of Dentistry and Oral Surgery, Tochigi National Hospital, ${ }^{8}$ Department of Oral and Maxillofacial Surgery, Dokkyo Medical University School of Medicine, ${ }^{9}$ Department of Stomatology and Oral Surgery, Gunma University Graduate School of Medicine, ${ }^{10}$ Kanagawa Dental College, Department of Oral and Maxillofacial Surgery, ${ }^{11}$ Department of Oral and Maxillofacial Surgery, Jichi Medical University, ${ }^{12}$ Department of Oral and Maxillofacial Surgery, Institute of Clinical Medicine, University of Tsukuba, ${ }^{13}$ Oral and Maxillofacial Oncology, Maxillofacial Diagnostic and Surgical Sciences, Faculty of Dental Science, Kyushu University, ${ }^{14}$ Department of Oral and Maxillofacial Surgery, Division of Medicine, Interdisciplinary Graduate School of Medicine and Engineering, University of Yamanashi

For setting of the diagnostic criterion value of dry mouth using an oral moisture checking device, we measured the moisture degree of tongue of 204 dry mouth patients and 241 healthy adults in 13 institutions and performed statistical analysis. Results: The moisture degree of tongue was $27.0 \pm 0.4$ in dry mouth group, and that was $29.6 \pm 0.2$ in healthy group. There was significantly different between two groups (Area under the curve; 0.659). However, the moisture degrees of buccal mucosa in dry mouth group and healthy group were $30.9 \pm 0.3$ and $31.3 \pm 0.2$ respectively, and the difference was not statistically significant.

\section{B-02. Salivary flow rate in healthy individuals}

Imamura $\mathrm{T}$, Yoshino $\mathrm{Y}$, Yamachika S, Kamoi M, Okamoto M, Yamamoto K, Kadomatsu S, Saito I, Nakagawa Y

Dry Mouth Clinic, Tsurumi University Dental Hospital

The purpose of this study was to examine the salivary flow rate in healthy individuals. The saliva samples from 252 dental hygienist students were analyzed. Resting (unstimulated) and stimulated salivary flow (gum test) was $5.2 \pm 3.3 \mathrm{~mL} / 15 \mathrm{~min}$ (ranging from 0.6 to 16.6 ) and $19.2 \pm 6.6$ $\mathrm{mL} / 10 \mathrm{~min}$ (ranging from 5.3 to 37.2 ), respectively. A $7.0 \%$ of the subject showed resting saliva flow less than
$1.5 \mathrm{~mL} / 15 \mathrm{~min}$. The study demonstrated that an individual difference was seen in the salivary secretion, and prevalence of salivary dysfunction was suggested to be higher than ever thought.

2B-03. A study of superlong-Term administration of cevimeline hydrochloride hydrate to xerostomia associated with Sjögren's syndrome

Iwabuchi H, Iwabuchi E, Uchiyama K, Fujibayashi T

Department of Dentistry and Oral Surgery, Tochigi National Hospital

The efficacy and adverse reactions of cevimeline hydrochloride hydrate were retrospectively evaluated in patients treated for superlong-Term administration. The study involved 118 patients continuously treated with this drug for 2 years or longer. The amount of saliva, subjective symptoms and adverse reactions were assessed during 5 years after treatment. Abnormal values of hematological parameters were noted in 16 patients 2 years or more after treatment. The amount of saliva reached a peak by 3.5 years from the start of treatment and tended to slowly decrease thereafter. Subjective symptoms showed maximum alleviation after one year, but mild symptoms were noted thereafter.

2B-04. Clinical evaluation of anti-centromere antibody in patients with Sjögren's syndrome

Kitagawa $\mathrm{T}^{1,2,3}$, Toya $\mathrm{S}^{2,3}$, Toyosima $\mathrm{K}^{2,3}$, Yamaguchi $\mathrm{A}^{2}$, Mori $\mathrm{K}^{4}$, Mataga $\mathrm{I}^{3,4}$, Shibasaki $\mathrm{K}^{1}$

${ }^{l}$ Course of Clinical Examination, Oral and Maxillofacial Surgery and Systemic Medicine, Graduate School of Life Dentistry at Niigata ${ }^{2}$ Oral and Maxillofacial Surgery ${ }^{3}$ Dry Mouth Clinic ${ }^{4}$ Department of Oral and Maxillofacial Surgery ,The Nippon Dental University

Anti-centromere antibody (ACA) has been reported to be associated with Sjögren's syndrome (SS) but the clinical significance of ACA remains unknown. We classified SS according to three autoantibodies (SS-A/Ro, SS-B/La, ACA) and assessed the clinical significance of ACA by comparing the clinical features of each group. Fifty-nine women patients diagnosed as SS in our Dry mouth clinic were enrolled in this study. Macroscopic findings of oral cavity and the amount of salivary excretion were evaluated. The clinical characters of ACA group were more serious than SS-A/Ro group and were almost equivalent to SS-B/La group.

\section{B-05. Two cases of Sjogren's syndrome patients in men}

Miyazaki $\mathrm{H}^{1}$, Inoue $\mathrm{Y}^{1}$, Izumisawa $\mathrm{M}^{1}$, Kubota $\mathrm{N}^{2}$, Fujibayashi $\mathrm{T}^{1}$, Kubota $\mathrm{E}^{1}$

${ }^{1}$ Department of Maxillofacial Surgery, Kanagawa dental College ${ }^{2}$ Department of Diagnostic Pathology, Kanagawa dental College Hospital

Prevalence rate of Sjogren's syndrome (SS) is supposed to be high in female rather than male, and the ratio is approximately 1 to 10 . Among 232 patients who suffered from xerostomia, we have diagnosed 25 female and 2 male patients as SS. We report these 2 SS cases in men according 
to the classification criteria in 1999 . These two male patients were both serologically negative. Saliva excretion rates and salivary function on scintigram were deteriorated. Biopsied lip glands showed lymphocyte infiltration with Focus score of 1 and 3 , respectively. We discuss on differential diagnosis of these two male SS cases.

2B-06. A case of IgG4-related disease (Mikulicz disease) with swelling of bilateral parotid, submandibular glands and lacrimal Gland

Mori $\mathrm{K}^{1}$, Toya $\mathrm{S}^{2}$, Fukushima $\mathrm{T}^{1}$, Mataga $\mathrm{I}^{1}$, Okada $\mathrm{Y}^{3}$, Toyama $\mathbf{M}^{4}$

${ }^{I}$ Department of Oral and Maxillofacial Surgery, The Nippon Dental University School of Life Dentistry at Niigata.

${ }^{2}$ Dry Mouth Clinic, Department of Oral and Maxillofacial Surgery, Niigata Hospital, The Nippon Dental University.

${ }^{3}$ Department of Oral Pathology, The Nippon Dental University School of Life Dentistry at Niigata.

${ }^{4}$ Department of Dental Radiology, The Nippon Dental University School of Life Dentistry at Niigata

Mikulicz disease is featured by swelling of salivary and lacrimal glands. Recently, this disease was putted on a new classification, IgG 4-related disease, independent from Sjögren's syndrome. We will report a case of IgG 4-related disease, Mikulicz disease. Patient was a 51 years old Japanese man who visited us at January 2010 with bilateral parotid, submandibular and lacrimal glands. He was also diagnosed as autoimmune pancreatitis by some medical examinations. Anti SS-A and anti SS-B antibodies in serum were negative, and serum IgG 4 was high, $1610 \mathrm{mg} / \mathrm{dl}$. IgG 4-positive plasmacyte infiltrations in salivary gland were observed from labial gland biopsy.

2B-07. Clinicopathological study on oral leukoplakia in patients who underwent curative surgical resection

Kuribayashi Y, Morita K, Yoshimoto M, Matsumoto K, Tsushima F, Sato M, Omura K

Oral and Maxillofacial Surgery, Graduate School of Medical and Dental Sciences, Tokyo Medical and Dental University

Various treatment procedures for oral leukoplakia have been reported. However, after some treatments, oral leukoplakia show recurrence. It has been reported that the rate of recurrence was 7.7-38.1\%.

A total of 41 oral leukoplakias that underwent curative surgical resection between 2004 and 2009 were studied. Comparisons with the rate of recurrence and clinical outcome, epithelial dysplasia, location, and resection margin were examined.

The rate of recurrence in curative surgical resection was $17 \%$. The common site of recurrence was gingiva, and closed resection margins $(<2 \mathrm{~mm})$ were observed in many recurrent cases. Recurrences have little association with a degree of epithelial dysplasia.
2B-08. Tobacco associated oral white lesions and oral submucous fibrosis - A Nepalese experience Prashanta Shrestha

Division of Oral and Maxillofacial Surgery, B and B Hospital, Kathmandu, Nepal

There is a country-dependent variation in habit factors associated with potentially malignant diseases of the oral mucosa. At least half of our patients presenting with squamous cell carcinoma, $(n=150)$ had tobacco quid habits and carcinoma exactly at the site where they frequently held the quid and one fourth of them had a pre existing oral submucous fibrosis. All patients presenting with submucous fibrosis $(n=120)$ were areca nut chewers. The 5-year survival rate, treated with surgery and radiation $(n=32)$, was 50 percent. The post treatment oral fibrosis was more severe in patients with oral submucous fibrosis, there by affecting their quality of life.

\section{B-09. A case of white sponge nevus with keratin 13 gene mutation}

Uka $\mathrm{M}^{1}$, Tsunoda $\mathrm{K}^{1}$, Kato $\mathrm{S}^{1}$, Kodaka $\mathrm{R}^{1}$, Tsunoda $\mathrm{H}^{1}$, Ouchi $\mathrm{T}^{2}$, Ishiko $\mathrm{A}^{2}$, Nakagawa $\mathrm{T}^{1}$

${ }^{1}$ Departments of Dentistry and Oral Surgery and ${ }^{2}$ Dermatology, Keio University School of Medicine

White sponge nevus (WSN) is a rare autosomaldominant inherited form of mucosal leukokeratosis. A 28-year-old male was determined to have unexplained oral white lesions since childhood despite oral biopsy. In 2008, his daughter was born and had the similar symptom in oral mucosa as proband. White lesion of pan oral mucosa was seen in both patients. Biopsy showed intracellular edema at the upper part of mucosal epithelium. Electron microscopy revealed aggregation of keratin filaments. Sequencing analysis of the proband revealed mutation of the mucosal keratin 13 gene. From these results, this patient was diagnosed as WSN.

\section{B-10. A case of suspected of intra oral pemphigus vegetans}

Koike $\mathrm{A}^{1}$, Miyashita $\mathrm{H}^{1}$, Takamori $\mathrm{K}^{1}$, Kimura $\mathrm{A}^{1}$, Tsunoda $\mathrm{K}^{2}$, Ikeuchi $\mathrm{S}^{1}$

${ }^{1}$ Oral Surgery, Shizuoka City Shimizu Hospital

${ }^{2}$ Department of Dentistry and Oral Surgery, Keio University School of Medicine

Pemphigus vegetans is an uncommon variant of pemphigus vulgaris. We report a case of pemphigus vegetans localized in buccal mucosa. A 74-year female noticed erosion on buccal mucosa. When teeth around erosion were extracted, erosion had worsened. Thus the patient was referred to our clinic for evaluation. Biopsy was performed from erosion. No definitive histopathological diagnosis was obtained. Since vegetation was observed, we suspected the lesion as autoimmune bullous diseases such as pemphigus vegetans. Desmoglein ELISA revealed positive against both Dsg3 and Dsg1. However no skin lesions were detected. Thus we suspected this patient as pemphigus vegetans localized in oral mucosa. 


\section{B-11. A case of pemphigus vulgaris suspected nickel allergy}

Naganawa K, Matsubara M, Sumitomo S, Shikimori M

Department of Oral and Maxillofacial Surgery, Division of Oral Pathogenesis and Treatment, Asahi University School of Dentistry

A case of pemphigus vulgaris with nickel allergy was presented. A 34 year-old woman referred our department, complaining haphalgesia in her mouth for 8 month. Erosions of whole gingival margin were observed. Steroid ointment was prescribed but subjective symptom went into a decline. Nickel allergy was revealed by patch test and metal prostheses were removed, but symptoms were not improvement. Both acantholysis in the biopsy specimen and higher antidesmogrein 3 antibody (anti-D3) in the serum gave a diagnosis of pemphigus vulgaris. Steroid therapy was performed referred to the serum anti-D3 level. After oneyear, neither intra-oral nor dermatological symptoms were observed.

2B-12. A case report of anti-p200 (laminin $\gamma$-1) and anti-laminin-332 mucous membrane pemphigoid

Mitate $\mathrm{E}^{1}$, Nakao $\mathrm{Y}^{1}$, Kobayashi $\mathrm{I}^{2}$, Hashimoto $\mathrm{T}^{3}$, Kiyoshima $\mathrm{T}^{2}$, Sakai $\mathrm{H}^{2}$, Kawano $\mathrm{S}^{1}$, Tanaka $\mathrm{A}^{1}$, Maehara $\mathrm{T}^{1}$, Hayashida $\mathrm{J}^{1}$, Nakamura $\mathrm{S}^{1}$

${ }^{1}$ Section of Oral and Maxillofacial Oncology, Division of Maxillofacial Diagnostic and Surgical Sciences, Faculty of dental science, Kyushu University. ${ }^{2}$ Laboratory of Oral Pathology, Division of Maxillofacial Diagnostic and Surgical Sciences, Faculty of dental science, Kyushu University. 3Department of Dermatology, School of Medicine, Kurume University

Recently, anti-p200 (laminin $\gamma$-1) and anti-laminin-332 mucous membrane pemphigoids are known as autoimmune blistering diseases. A 61 year-old-man who was transplanted a living-donor kidney in June 2006 stopped taking immunosuppressant on his own judgment in January 2009. He had resulted in fever in that August, and was hospitalized of our internal medicine to find the origin of fever in that December. Also he has found blisters on his palate, buccal and ranine mucosae since that August. On the biopsy and serum findings, pemphigoid with anti-p200 and anti-laminin-332 autoantibodies is considered. The blisters diminished in re-taking the immunosuppressant.

2B-20. Expression and localization of arylalkylamine $\mathrm{N}$-acetyltransferase (AANAT) in rat salivary glands

Shimozuma $\mathrm{M}^{1}$, Tatehara $\mathrm{S}^{1}$, Mishima $\mathrm{K}^{2}$, Umeki $\mathrm{H}^{1}$, Saito $\mathrm{I}^{2}$, Satomura $\mathrm{K}^{1}$

${ }^{I}$ Second Department of Oral and Maxillofacial Surgery, Department of Oral Medicine and stomatology, School of Dental Medicine, Tsurumi University

${ }^{2}$ Department of Pathology, School of Dental Medicine, Tsurumi University

Melatonin is known to regulate a variety of physiological processes including control of circadian rhythms. Melatonin was proven to exist in saliva. In the present study, to clarify the origin of melatonin in saliva, the expression of arylalkylamine $\mathrm{N}$-acetyltransferase (AANAT) in rat salivary glands was immunohistochemically investigated. As a result, AANAT was noted to localize in ductal epithelial cells in salivary glands. RT-PCR analysis demonstrated the expression of mRNA for AANAT in submandibular gland. These findings demonstrated that melatonin might be produced and secreted by salivaly glands and suggested that melatonin might play a physiological role in the oral cavity.

\section{B-21. Extracellular ATP induces expression of MHC classII on human salivary gland ductal epithelial cells}

Itoh $\mathrm{A}^{1,2}$, Nagai $\mathrm{Y}^{2}$, Sugawara $\mathrm{Y}^{1}$, Sasano $^{1}$, Sugawara $\mathrm{S}^{2}$

${ }^{1}$ Division of Oral Diagnosis, Tohoku University Graduate School of Dentistry

${ }^{2}$ Division of Oral Immunology, Tohoku University Graduate School of Dentistry

Extracellular ATP (eATP) is important for cell-to-cell communication in the immune system. In this study, we examined the role of eATP in immunological response using a human salivary gland ductal epithelial cell line HSG. We stimulated HSG cells with high concentration ATP for 24 hours and examined MHC classII expression. After the stimulation, significant increase in the expression of MHC classII on cell membrane and in cytoplasm were detected by FACS and immunofluorescence analyses. In addition, BzATP, a P2X7receptor agonist, showed the same reaction. These results suggest that eATP participate in breaking peripheral tolerance through $\mathrm{P} 2 \mathrm{X} 7$ receptor.

\section{B-22. Clinical study of Glossodynia at our hospital} during the past five years

Okubo $\mathrm{M}^{1}$, Wakui $\mathrm{T}^{1}$, Kawamata $\mathrm{H}^{1}$, Imai $\mathrm{Y}^{1}$

${ }^{1}$ Department of oral and maxillofacial surgery, Dokkyo Medical University School of medicine

We report of clinical study on 177 cases of glossodynia, who treated at the department of oral and maxillofacial surgery, Dokkyo medical University School of medicine. The ratio of male to female was 1 to 5(29 males, 148 females), and mean age of patients were 58.5 years old. The most frequent site of pain was the apex of the tongue. Many patients had systemic disease (Cardiovascular disease:55 psychiatric disease: 28, gastrointestinal diseases: 17) For treatment, external medicine, SSRI, antianxiety drug, Chinese medicine were used. As for treatment of glossodynia, combination therapy was more effective than single therapy.

\section{Poster presentation}

\section{P-01. The character of lining epithelium of kerato- cystic odontogenic tumors}

Sakano Y, Itoh N, Nagayama M, Tanuma J

Department of Oral Pathology, Division of Oral Pathogenesis and Disease Control, Asahi University School of Dentistry

Keratocystic odontogenic tumor (KCOT) has characterized in a high tendency of recurrence mode when the 
lining epithelium and/or daughter cyst were left after surgical treatment. This study was focused on the basal layer showing their cellular proliferation used by the immunohistochemical detection of Ki-67.

In the results, Ki-67 positive cells of KCOT lining epithelium corresponded to their morphological change such as parakeratinization, palisading of basal layer and epithelial budding.

\section{P-02. Perlecan-binding growth factors in kerato- cystic odontogenic tumors}

Hasegawa $\mathrm{M}^{1,2}$, Cheng $\mathrm{J}^{1}$, Maruyama $\mathrm{S}^{3}$, Yamazaki $\mathrm{M}^{1}$, Saito $\mathrm{C}^{3}$, Saku $\mathrm{T}^{1,3}$

Divisions of ${ }^{1}$ Oral Pathology, ${ }^{2}$ Reconstructive Surgery for Oral and Maxillofacial Region, Niigata University Graduate School of Medical \& Dental Sciences, ${ }^{3}$ Oral Pathology Section, Department of Surgical Pathology, Niigata University Hospital

We have already demonstrated the intercellular deposit of perlecan in the epithelial lining of keratocystic odontogenic tumors (KCOTs). To study further functions of perlecan in this particular epithelial zone, we analyzed 20 surgical samples of KCOT for perlecan-binding growth factors by immunohistochemistry. Perlecan, E-cadherin, and $\beta$-catenin were localized on the cell border of the cyst lining. KGF was also on the cell border in addition to the cytoplasm in the same zone, while ?-dystroglycan on the cell membrane was limited to the parabasal and middle zones. VEGF and shh were localized in the lower half of the epithelium, where Ki-67 was labeled. It is thus suggested that perlecan-bound molecules function in cell proliferation as well as in cell differentiation.

\section{P-03. Expression of tenascin in dentinogenic ghost cell tumor}

Matsuoka $\mathrm{K}^{1}$, Matsuzaka $\mathrm{K}^{1}$, Hara $\mathrm{A}^{2}$, Kokubun $\mathrm{K}^{2}$, Nakajima $\mathrm{K}^{1}$, Kimura $\mathrm{Y}^{1}$, Hashimoto $\mathrm{K}^{1}$, Miwa $\mathrm{T}^{1}$, Muramatsu $\mathrm{T}^{2}$, Hashimoto $\mathrm{S}^{2}$, Shimono $\mathrm{M}^{2}$, Inoue $\mathrm{T}^{1}$ Department of Clinical Pathophysiology ${ }^{l}$, Tokyo Dental College Department of Pathology ${ }^{2}$, Tokyo Dental College

DGCT is characterised by ghost cell, dentinogenic calciyfing tissue, and ameloblastoma-like epithelial tissue. We evaluated the expression of Tenascin in DGCT in terms of tumorgenesis using immunohistochmical staining. Type I collagen, Fibronectin, CK13, and CK19 were also used as primary antibody, and Congo red for special staining. Tenascin was expressed beneath the basement membrane of epithelial component of DGCT, just same manner as ameloblastoma. Furthermore Tenascin was expressed in the dentinogenic calcified tissue. These results suggest that tumorgenesis of DGCT is similar to ameloblastoma, but odontogenic mesenchymal cells might be existed in the DGCT and differentiated into odontoblast by Tenascin.
P-04. A case of calcifying cystic odontogenic tumor: Immunohistochemical examination of Notch

Nakano $\mathrm{K}^{1}$, Tsujigiwa $\mathrm{H}^{2}$, Nagatsuka $\mathrm{H}^{2}$, Hasegawa $\mathrm{H}^{1}$, Kawakami $\mathrm{T}^{1}$

${ }^{1}$ Department of Oral Pathology, Matsumoto Dental University School of Dentistry

${ }^{2}$ Department of Oral Pathology and Medicine, Okayama University Graduate School of Medicine, Dentistry and Pharmaceutical Sciences

The epithelial and mesenchymal interaction is directly reflects the development of odontogenic neoplasms. Therefore, we have examined Notch signaling in a case of calcifying cystic odontogenic tumor (CCOT), with ameloblastic fibroma and odontogenic myxoma cases as controls. In these specimens, Notch-positive-products were present both in the epithelial and ectomesenchymal components in the CCOT and the ameloblastic fibroma case, but negative in the odontogenic myxoma case, having no odontogenic epithelial islands. The examination results suggest that Notch signaling plays some important roles in cytological differentiation or acquisition of tissue-specific characteristics between odontogenic epithelium and odontogenic ectomesenchymal tissues.

\section{P-05. Expression of Runx 2 in ameloblastomas}

Ishida $\mathrm{K}^{1,2}$, Usami $\mathrm{Y}^{3}$, Kishino $\mathrm{M}^{1}$, Sato $\mathrm{S}^{1}$, Ogawa $\mathrm{Y}^{1}$, Ikebe $\mathrm{K}^{2}$, Toyosawa $\mathrm{S}^{1}$

${ }^{1}$ Department of Oral Pathology, ${ }^{2}$ Department of Prosthodontics and Oral Rehabilitation, Osaka university Graduate School of Dentistry, ${ }^{3}$ Clinical Laboratory, Osaka University Dental Hospital

Ameloblastoma with high alkaline phosphatase activity in the stromal cells and some cases of stromal ossification has been reported. These findings suggest that the stromal cells of ameloblastoma has osteogenic property. In this study, we investigated the immunohistochemical distribution of Runx2-positive cells, a marker of osteoprogenitor cells, in ameloblastoma using anti-Runx2 antibody. As a result, Runx 2 expression was detected on the stromal cells just below the epithelial component of ameloblastoma, but not of inflammatory non-odontogenic cyst. It was speculated that epihtelial component of ameloblastoma could induce the differentiation of the surrounding stromal cells into osteoprogenitor cells.

\section{P-06. Immunohistochemical study of tumor prop-} erty of unicystic ameloblastoma

- comparison with lining epithelium of dentigerous cyst -

Ohkubo $\mathrm{Y}^{1}$, Kanri $\mathrm{Y}^{1}$, Okada $\mathrm{Y}^{1,2}$

${ }^{1}$ Department of Pathology, The Nippon Dental University, School of Life Dentistry at Niigata

${ }^{2}$ Clinical Laboratory, The Nippon Dental University Niigata Hospital

Unicystic ameloblastoma is histopathologically characterized by unilocular formation and involves impacted third molar tooth of mandible in many cases. In the diagnosis of unicystic ameloblastoma, it is important to distinguish it 
from dentigerous cyst. We conducted immunohistochemical study on 5 cases of unicystic type among 21 cases recently diagnosed as ameloblastoma in our department and clinical laboratory, using CK10, CK13, CK17, CK19, calretinin, bcl-2, Bax, Ki67, p53, and Wnt-1. We compared the results of the immunohistochemical staining between unicystic ameloblastoma and lining epithelium of dentigerous cyst to investigate tumor property of unicystic ameloblastoma.

\section{P-07. A case of mandibular ameloblastoma demon- strating a papillary growth in the gingiva}

Yamamura $\mathrm{K}^{1}$, Tanaka $\mathrm{Y}^{2}$, Yamashina $\mathbf{M}^{1}$, Tonogi $\mathbf{M}^{1}$, Yamane $\mathrm{G}^{1}$

${ }^{1}$ Department of Oral Medicine, Oral and Maxillofacial Surgery, Tokyo Dental College

${ }^{2}$ Division of Clinical Laboratory, Ichikawa General Hospital, Tokyo Dental College

Ameloblastoma is a relatively common tumor of the mandible, and peripheral ameloblastoma is sometimes known to cause a papillary growth in the surrounding gingiva. It is, however, extremely rare for an intraosseous ameloblastoma to extend into the covering mucosa and thereby induce a papillary growth in the gingiva. The rare type of ameloblastoma, if encountered, may clinically require differential diagnosis against malignant tumor. We have experienced a case of a 36-year-old man who presented intraosseous mandibular ameloblastoma with papillary growth in the gingival epithelium.

\section{P-08. Fate of epithelial cell rests of Malassez on MNU-induced rat molar regions}

Kawanaka A, Yoshizawa K, Rai C, Miki H, Kuwata M, Kanematsu S, Uehara H, Yuri T, Tsubura A

\section{Department of Pathology II, Kansai Medical University}

Epithelial cell rests of Malassez becomes the epithelial lining of an odontogenic cyst due to the active inflammation, and it also forms the odontogenic tumor. However, the detailed mechanisms are unknown. Female Lewis rats were treated i.p. with $50 \mathrm{mg} / \mathrm{kg} \mathrm{N}$-methyl-N-nitrosourea (MNU) at 4 weeks of age. At the age of 18 weeks, rats were sacrificed to sample maxilla and mandible areas with $H \& E$ staining. Epithelial cells in molar region nests were counted in each unit area. The MNU-treated group showed greater number of epithelial cells in each nest compared with the controls. In addition, mandible had higher incident of an epithelial nests than maxilla. As in both area, $80 \%$ of the nests were located at cervical and furcation area of M2 and M3. The histological result correlates with site of predilection for ameloblastoma.

\section{P-09. Characterization of cartilage-like element in pleomorphic adenoma}

Kokubu $\mathrm{M}^{1}$, Tominaga $\mathrm{K}^{2}$, Masuno $\mathrm{K}^{2}$, Wato $\mathrm{M}^{2}$, Nishikawa $\mathrm{T}^{2}$, Tanaka $\mathrm{A}^{2}$

${ }^{I}$ Graduate School of Dentistry (Pathology), ${ }^{2}$ Department of Oral Pathology, Osaka Dental University

Pleomorphic adenoma is the most common tumor of the salivary glands, and shows cytomorphologic and architectural diversity. Histopathologically, this neoplasm involves cartilage-like element at frequency of several to 20 percent, and chondrocyte-like cells are involved in the cartilage lacuna of the basophilic matrix. The aim of the present study was to characterize the cartilage-like element. Formalinfixed, paraffin-embedded sections $(n=5)$ were stained with toluidine blue and immunostained with anti-type II collagen antibody, anti-Sox9 antibody, and anti- VIII factor antibody. Although metachromasia was found in the most sections of cartilage-like element, type II collagen was not detected in almost all the elements. Sox 9 was positive in the chondrocytelike cells, and factor VIII was negative. Therefore, the cartilage-like element is similar to cartilage, but does not seem to be true cartilage.

\section{P-10. Emil siRNA enhances the induction of apop- tosis by anticancer agent}

Shimizu N, Kudo Y, Tsunematsu T, Takata T

Department of Oral and Maxillofacial Pathobiology, Hiroshima University Graduate School of Biomedical Sciences

Emi1 is known to have the function to inhibit anaphase promoting complex (APC), which is a cell cycle-regulated ubiquitin ligase complex. It recently has been shown that Emi1 knockdown prevents transition from $\mathrm{S}$ to $\mathrm{G} 2$ phase by downregulating geminin through the activation of APC. Most of anticancer agents prevent the separation of the two strands during DNA replication. Therefore, we thought that Emi1 knockdown might enhance the sensitivity for anticancer agents. First we confirmed that Emi1 siRNA induced polyploidy for preventing transition from $S$ to $G 2$ phase in several cancer cell lines. Next, we treated adriamycin in Emi1 siRNA treated cancer cells. Interestingly, increased apoptotic cells were observed after treatment with anticancer agents in Emi1 knockdown cells. These results suggest that inhibition of Emil could be a useful tool for enhancing of sensitivity of cancer cells to anticancer agents.

\section{P-11. Spindle cell carcinoma in lower gingival. Report of a case}

Kitamura $\mathrm{T}^{1}$, Higashino $\mathrm{F}^{1}$, Abiko $\mathrm{Y}^{2}$, Shindoh $\mathrm{M}^{1}$

${ }^{1}$ Department of Oral Pathology and Biology, Hokkaido University Graduate School of Dental Medicine

${ }^{2}$ Division of Oral Medicine \& Pathology, Department of Dental Science, Institute of Personalized Medical Science, Health Science University of Hokkaido

We report a case of spindle cell carcinoma in lower gingiva. A 43-year-old female consulted Hokkaido Health Science University Hospital with a chief complaint of deflection of the right lower first molar. Clinical diagnosis of marginal periodontitis was made and followed up. Three months later from the clinical onset, she manifested swelling and parenthesis of right mandible. Biopsy specimen revealed spindle cell carcinoma. She was introduced to Hokkaido University Hospital, and tumor resection was carried out.

Pathological examination showed that spindle shaped atypical tumor cells proliferated with mandibular bone destruction She is in disease-free condition till now. 
P-12. Evaluation of delayed cervical lymph node metastasis in stage I/II squamous cell carcinoma of the tongue

Matayoshi A, Sunakawa H

Department of Clinical Neuroscience, Oral and Maxillofacial Functional Rehabilitation, Graduate School of Medicine, University of the Ryukyus

\section{Purpose}

The purpose of current study was to predict the risk of delayed cervical lymph node metastasis (DCLNM) in stage I/II squamous cell carcinoma of the tongue (SCCT).

\section{Methods}

This study concerns 54 patients with T1/2 TSCC. Clinical characteristic, histopathological parameter, depth of invading to muscle layer, intratumoral lymphatic vessel density (LVD) and intratumoral microvessel density (MVD) were evaluated.

\section{Results}

Deep invasion group and high LVD group were associated with metastasis.

\section{Conclusion}

Evaluating depth of muscle invasion and LVD was useful for predicting DCLNM in stage I/II TSCC.

P-13. Detection of HPV and expression of related genes in epithelial dysplasias and squamous cell carcinomas of the oral mucosa

Tsuji $\mathrm{K}^{1,2}$, Wato $\mathrm{M}^{3}$, Kamitani $\mathrm{M}^{1}$, Iwamoto $\mathrm{S}^{1}$, Iseki $\mathrm{T}^{2}$, Shima $\mathrm{M}^{1}$, Tanaka $\mathrm{A}^{3}$, Morita $\mathrm{S}^{2}$

${ }^{1}$ Oral Surgery, Kishiwada City Hospital

${ }^{2}$ First Department of Oral and Maxillofacial Surgery, Osaka Dental University

${ }^{3}$ Department of Oral Pathology, Osaka Dental University

We examined to clarify the detection of HPV and related genes in oral dysplasias and oral squamous cell carcinomas. We studied detection of HPV DNA by PCR and immunohistochemical expression of p53, Rb, p16, p21 and CyclinD1. HPV 16 was detected almost in CIS, WSCC and PSCC. Remarkable expression of $\mathrm{p} 53, \mathrm{Rb}$ and CyclinD1 was found in CIS, WSCC and PSCC. p21 was negative in CIS, WSCC and PSCC. These results suggest that HPV16 may play an important role in the transformation of p53 and Rb.

\section{P-14. Two cases of adenoid cystic carcinoma of the tongue with a chief complaint of glossalgia}

Satoh $\mathrm{J}^{1}$, Hatakeyama $\mathrm{S}^{1}$, Yodogawa $\mathrm{S}^{2}$, Ohnishi $\mathrm{T}^{2}$, Kaku $\mathrm{T}^{1}$, Nagayasu $\mathrm{H}^{2}$, Abiko $\mathrm{Y}^{1,3}$

${ }^{I}$ Graduate School of Dentistry, Health Sciences University of Hokkaido

${ }^{2}$ Department of Oral Surgery, Institute of Personalized Medical Science, Health Sciences University of Hokkaido

${ }^{3}$ Department of Dental Science, Institute of Personalized Medical Science,Health Sciences University of Hokkaido

We report two cases of adenoid cystic carcinoma (ACC) in the tongue. Case 1: A 59-year-old man was admitted to our hospital complaining of symptoms consistent with glossalgia. The patient was diagnosed as having burning mouth syndrome (BMS) at two other hospitals. The elastic hard swelling was present in the sublingual region. Case 2: A 60-year-old Japanese woman with the same complaint as the above case. A rice-grain sized tumor was present in the sublingual region. Both cases were diagnosed as ACC. Malignant tumor cannot be ruled out even if the surface is covered with intact epithelium.

\section{P-15. A case of histiocytic sarcoma in the maxillary gingiva}

Ochiai $\mathrm{T}^{1}$, Aizawa $\mathrm{S}^{2}$, Kimura $\mathrm{A}^{1,2}$, Nakano $\mathrm{K}^{1,2}$, Kawakami $\mathrm{T}^{1,2}$, Hasegawa $\mathrm{H}^{1,2}$

${ }^{1}$ Department of Oral Pathology, Matsumoto Dental University

${ }^{2}$ Hard Tissue Pathology Unit, Matsumoto Dental University Graduate School

Histiocytic sarcoma is a rare neoplasm which is usually aggressive tumor with a poor response to therapy. We report a case of histiocytic sarcoma arising from maxillary gingiva. A 71-year-old male was referred to our hospital for gingival region gradually swelling and bleeding. Microscopical examinations revealed that the tumor was composed histiocyticatypicalcellproliferation.Immunohistochemically, tumor cells were positive for EMA, vimentin, LCA and CD68, and negative for AE1/AE3, S100, CD21, CD30, CD56, CD1a, CD3 and CD20. Ki-67 labeling index was $38 \%$, accompanied with positive reaction to $\mathrm{p} 53$.

\section{P-16. MTX associated lymphoproliferative disorder of the oral cavity in a patient with rheumatoid arthritis}

Kikuchi $\mathrm{K}^{1}$, Inoue $\mathrm{H}^{1}$, González-Alva $\mathrm{P}^{1}$, Miyazaki $\mathrm{Y}^{1}$, Tanaka $\mathrm{A}^{2}$, Shigematu $\mathrm{H}^{2}$, Ide $\mathrm{F}^{1}$, Yamauchi $\mathrm{M}^{3}$, Sakashita $\mathrm{H}^{2}$, Kusama $\mathrm{K}^{1}$

${ }^{1}$ Division of Pathology, ${ }^{2}$ Division of Second Oral and Maxillofacial Surgery Department of Diagnostic and Therapeutic Sciences, ${ }^{3}$ Division of Medical Informatics Department of Community Health Sciences, Meikai University School of Dentistry

Patients affected by autoimmune diseases who are treated with methotrexate (MTX) sometimes develop lymphoproliferative disorders (LPDs). In approximately $40 \%$ of reported cases, the affected sites are extranodal, and are included the gastrointestinal tract, skin, lung, kidney, and soft tissues. However, MTX-associated LPD (MTX-LPD) is extremely rare in the oral cavity. Here we report a 69-yearold Japanese woman with rheumatoid arthritis (RA) who developed MTX-LPD resembling Hodgkin's disease (Hodgkin lymphoma-like lesion; HL-like lesion) in the left upper jaw. Histopathologically, large atypical lymphoid cells including Hodgkin or Reed-Sternberg-like cells were found to have infiltrated into granulation tissue in the ulcerative oral mucosa. Immunohistochemistry showed that the large atypical cells were positive for CD20, CD30 and EpsteinBarr virus (EBV)-latent infection membrane protein-1 (LMP-1) and negative for CD15. EBV was detected by in situ hybridization (ISH) with EBV-encoded small RNA (EBER), and polymerase chain reaction (PCR) for LMP-1 and EBNA-2 in material taken from the formalin-fixed, paraffin-embedded specimen. 


\section{P-17. Correlation between candy test and whole saliva flow rate in healthy young adults}

Kurihara M, Yamamoto K, Matsusue Y, Komatsu Y, Nakagawa Y, Matsubara Y, Imanishi M, Tsuyuki M, Kirita T

Department of Oral and Maxillofacial Surgery, Nara Medical University

Correlation between candy test (CT) and whole saliva flow rate was investigated in 313 healthy young adults. Whole saliva was collected under unstimulated condition (UT) and stimulated condition by gum test (GT) and Saxon test (ST). CT was performed according to the method of Wolff with modification. The average of UT, GT and ST was significantly higher in males and correlated moderately one another. CT was only weakly correlated with GT and ST in females or in whole subjects. These results indicate CT cannot be the alternative method of whole saliva flow rate in the present form.

P-18. Correlation between whole saliva flow rate and occlusal force in healthy young adults

Komatsu Y, Yamamoto K, Matsusue Y, Kurihara M, Nakagawa Y, Matsubara Y, Imanishi M, Tsuyuki M, Kirita T

Department of Oral and Maxillofacial Surgery, Nara Medical University

Correlation between whole saliva flow rates under unstimulated condition (UT) or stimulated condition by gum test (GT) and Saxon test (ST) and body profiles such as height and weight and occlusal area (OA), occlusal pressure (OP) and occlusal force (OF) were analyzed in 52 young adults. The averages of UT, GT and ST were $5.36 \mathrm{ml} / 10 \mathrm{~min}$, $23.18 \mathrm{ml} / 10 \mathrm{~min}$ and $4.91 \mathrm{~g} / 2 \mathrm{~min}$, respectively, and those of $\mathrm{OA}$, OP and OF were $19.3 \mathrm{~mm}^{2}, 41.5 \mathrm{MPa}$ and $758.8 \mathrm{~N}$, respectively. Weak correlation of whole saliva flow rates with body profiles was suggested but not either with OA, OP or OF.

P-19. Clinical characteristics and treatment outcome in xerostomia clinic outpatients

Funayama $\mathrm{S}^{1}$, Ito $\mathrm{K}^{1}$, Inoue $\mathrm{M}^{1,2}$

${ }^{I}$ Geriatric Dentistry, Niigata University Medical and Dental Hospital

${ }^{2}$ Division of Dysphagia Rehabilitation, Niigata University Graduate School of Medical and Dental Sciences

The purpose of this study was to investigate clinical characteristics and treatment outcome in xerostomia clinic outpatients. Subjects were composed of 698 outpatients of xerostomia clinic at Niigata University Medical and Dental Hospital. Out of all patients, 33.5\% were diagnosed as neurogenic xerostomia, $15.6 \%$ were drug-induced xerostomia and $13.5 \%$ as Sjogren's syndrome. The $39.4 \%$ patients were finished their treatment. The mean period of treatment was 5.6 months. In addition, subjective symptom was improved in $79.0 \%$ of patients. The results demonstrate that the treatment of xerostomia may brought a good effect.
P-20. Clinical study of the bullous pemphigoid presented symptoms to the oral mucosa

Miyamoto S, Kaneko T, Yasuda T, Chikazu D

Department of Oral and Maxillofacial Surgery, Tokyo Medical University

Bullous pemphigoid (BP) is one of the most common of autoimmune blistering conditions, it occurs at the estimated rate 10 cases per million individuals per a year.

In general, BP is a chronic blistering disease of the skin, the oral mucous membranes were involved in approximately $8-40 \%$ of the patients.

We describe 5 uncommon cases of patients with BP that initially involved the oral mucosa.

Patients were diagnosed with BP on the basis of the results of direct pathological examinations in the biopsy specimens of the oral mucosa as well as the results of enzyme-linked immunosorbent assay of the BP180.

\section{P-21. Immunohistochemical analysis of immune response in oral lichen planus}

Oshita $\mathrm{Y}^{1}$, Kishino $\mathrm{M}^{1}$, Usami $\mathrm{Y}^{2}$, Sato $\mathrm{S}^{1}$, Ogawa $\mathrm{Y}^{1}$, Toyosawa $\mathbf{S}^{1}$

${ }^{1}$ Department of Oral Pathology, Osaka University Graduate School of Dentistry

${ }^{2}$ Clinical Laboratory. Osaka University Dental Hospital

Oral lichen planus (OLP) is a chronic inflammatory disease occuring typically on bilateral buccal mucosa, where it appears as either white reticular, plaque or erosive lesions. At present, the causative factors are still unknown. Histologically, OLP is characterized by band-like infiltrate of lymphocytes in the mucosal interface, epithelial hyperkeratosis, saw-tooth appearance and basal cell liquefaction degeneration. It is considered that the stimulated T-lymphocytes are guided toward the basement membrane and cause progression of the disease by keratinocyte expressing ICAM-1 and MHC-2.

In this study, we retrieved typical OLPs from pathology files of our hospital. Immunohistochemical stain was performed using following monoclonal antibodies: CD3, CD4, CD8, CD20. CD54(ICAM-1), CD68, CD79a, HLA-D.

P-22. Studies for the effect and safety of an antimycotic medicine on the patients complaining of intraoral pain

Harada $\mathrm{K}^{1}$, Niinaka $\mathrm{Y}^{2}$, Higuchi $\mathrm{M}^{2}$, Oda $\mathrm{M}^{2}$, Kawasaki $\mathrm{T}^{2}$, Yoshida $\mathrm{C}^{2}$, Nakazawa $\mathrm{R}^{2}$, Miura ${ }^{2}$, Dohi $\mathrm{A}^{2}$, Nakano $\mathrm{Y}^{3}$ ${ }^{1}$ Department of oral and maxillofacial surgery, Interdisciplinary graduate school of medicine and engineering, University of Yamanashi.

${ }^{2}$ Oral and maxillofacial surgery, University hospital, Faculty of medicine, University of Yamanashi.

${ }^{3}$ Oral implant center, University hospital, Faculty of medicine, University of Yamanashi

There are various causes for the intraoral pain. Oral fungus is one of such a cause. In this study, we examined the effect of the itraconazole internal liquid for intraoral pain. Subjects were 22 patients. Two hundred milligrams of the itraconazole were administered per day for one week. 
Mycological examination and check of the symptoms were performed before and after the administration. Candida albicans was detected in 7 patients before the administration. Intraoral pain reduced significantly after the administration. In conclusion, the itraconazole was suggested to be useful for improvement of the intraoral pain caused by oral fungus.

\section{P-23. The change of Candida species in oral cavity in the aged with denture}

Goto J, Yamazaki Y, Hata H, Murata T, Kitagawa Y

Oral Diagnosis and Oral Medicine, Department of OralPathob iologicalScience,Graduate School of Dental Medicine, Hokkaido University

Aim: To elucidate the effects of the dentures on changes of Candida species. Materials and Methods: We examined 391 subjects of advanced age (65-75 years).Samples for culture of Candida species were collected from dorsum linguae from all of the subjects. Seventy-six subjects, with dentures covering hard palate, received culture examination form hard palate.

Results: As for the Candida species, Candida albicans was the predominant species in subjects without dentures. The prevalence of non-albicans species and the plural species significantly increased in subjects with dentures.

Conclusions: This study suggests that denture have significant effects on changes of Candida species.

P-24. Clinical examination of the subjective symptom improvement by the antifungal drug to the oral candidiasis patients -the second report-

Watanabe K, Mese H, Ibaragi S, Sasaki A

Department of Oral and Maxillofacial Surgery, Okayama University Graduate School of Medicine, Dentistry and Pharmaceutical Sciencess

This study was performed to investigate the subjective symptom improvement by the antifungal drug to the oral candidiasis patients. The subjects were 112 patients examined in our hospital during a three year period from April 2007 to March 2010. They included thirty tow men and 80 women with a mean age 66.0 years and range of 21 to 89 years old. We classified subjective symptoms (pain, oral dysesthesia, dry mouth sensation and taste disturbance). As a result, 60 patients were pain, 23 patients were dysesthesia, 15 patients were dry mouth sensation and 14 patients were taste disturbance. The efficacy rates in the oral candidiasis patients treated with antifungal drug were $81.3 \%$.
P-25. Nicotinic acetylcholine receptor regulates periodontal destruction and inflammatory cytokines expression during experimental periodontitis in rats

Liu $\mathrm{YF}^{1,2}$, Miwa $\mathrm{T}^{2}$, Matsuoka $\mathrm{K}^{2}$, Kimura $\mathrm{Y}^{2}$, Matsuzaka $\mathrm{K}^{2}$, Inoue $\mathrm{T}^{2}$

${ }^{1}$ Department of Pediatric Dentistry, School of Stomatology, Fourth Military Medical University, Xi'an, China

${ }^{2}$ Department of Clinical Pathophysiology and Oral Health Science Center HRC 7, Tokyo Dental College

We investigated the effects of nicotine on the functional expression of $\alpha 7 \mathrm{nAChR}$ in rat periodontal tissues. The expression of $\alpha 7 \mathrm{nAChR}$ was up-regulated by nicotine administration, which chould be partially suppressed by alpha-bungarotoxin. The expressions of IL- $1 \beta$, IL-6 and TNF- $\alpha$ were correlated with $\alpha 7$ nAChR expression. Our study indicates that nicotine may affect periodontal tissue destruction and inflammatory cytokines expression through regulation of $\alpha 7 \mathrm{nAChR}$ in rat periodontal tissues.

P-26. Experimental study on relationship between periodontitis and systemic diseases -Systemic influence of continuous application of LPS to periodontal tissues -

Furusho $\mathrm{H}^{1}$, Aomin ${ }^{2}$, Inubushi $\mathrm{T}^{1}$, Miyauchi $\mathrm{M}^{1}$, Kitagawa $\mathrm{M}^{3}$, Takata $\mathrm{T}^{1}$

${ }^{1}$ Department of Oral and Maxillofacial Pathobiology, ${ }^{2}$ Department of Pediatric Dentistry, ${ }^{3}$ Center of Oral Clinical Examination, Hiroshima University

Chronic low-grade inflammation is an important modulator of systemic diseases like cardiovascular disease and diabetes. We examined the effects of continuously applied-LPS into a pulp chamber on periapical periodontal tissue and distant organs including adipose tissue, liver, aorta etc. An acute periapical lesion was developed at 4-week-application and a shift from acute to chronic inflammation was evident at 6-week-application. In LPSapplied animals, LPS was detected in serum and epididymal fat pad gradually increased. The slight pathological changes and upregulation of inflammatory cytokines were observed in the organs examined. The results suggest that LPS into blood circulation from periapical periodontal tissues may induce distant organ injury.

\section{P-27. Butyrate upregulates expression of $\beta$-defensins in keratinocytes}

Yamazaki $\mathrm{M}^{1}$, Nishimura $\mathrm{M}^{1}$, Uemura $\mathrm{T}^{1}$, Kaku $\mathrm{T}^{1}$, Abiko $\mathrm{Y}^{1,2}$

Division of Clinical Oral Pathology, Health Sciences University of Hokkaido ${ }^{1}$

Division of Oral Medicine \& Pathology, Institute of Personalized Medical Sciences, Health Sciences University of Hokkaido ${ }^{2}$

Human beta-defensins (hBDs) are innate antimicrobial peptides produced in keratinocytes, which play an important role in the protective barrier of oral epithelium. Butyrate, the dietary histone deacetylase inhibitor, is produced by periodontal pathogens such as porphyromonas gingivalis and fusobacterium nucleatum. The present study investigated 
how butyrate affected the expression patterns of hBD-2 and -3 in HaCaT keratinocytes. Butyrate induced upregulated expression of hBDs at both mRNA and protein levels. The JNK II and the AG1478 inhibited butyrate-induced JNK and ERK activation. These results suggest that butyrate induces upregulated expression of hBDs in the keratinocyte, and its possibly involved in MAPK-pathway.

\section{P-28. A case of benign fibrous histiocytoma of the buccal mucosa}

Oyazato $\mathrm{Y}^{1}$, Hirano $\mathrm{M}^{1}$, Semba $\mathrm{I}^{1}$, Nozoe $\mathrm{E}^{2}$, Nakamura $\mathrm{N}^{2}$

${ }^{1}$ Department of Oral Pathology, Graduate school of Medical and Dental Sciences, Kagoshima University.

${ }^{2}$ Department of Oral and Maxillofacial Surgery, Graduate school of Medical and Dental Sciences, Kagoshima University

Benign fibrous histiocytoma is a soft tissue tumor arising frequently in the skin, however it is rare in the oral mucosa.

A 39-year-old female was referred our hospital for evaluation and treatment of a mass in the right buccal mucosa. Clinical examinations disclosed the submucosal mass in $1.0 \mathrm{~cm}$ diameter. An excision of the mass was made.

Histologically, the tumor was composed of irregular arranged dense proliferation of short spindle cells having ovoid nucleus with nucleoli and without mitotic figure. Immunohistochemically, the tumor cells were positive for vimentin, CD68 and Factor XIIIa and Ki-67 index was less than $3 \%$.

\section{P-29. Nerilemmoma in floor of mouth diagnosed} with fine needle aspiration cytology: a case report Muramatsu $\mathrm{T}^{1}$, Hashimoto $\mathrm{S}^{1}$, Yamaguchi $\mathrm{A}^{1}$, Hara $\mathrm{A}^{1}$, Matsuzaka $\mathrm{K}^{2}$, Inoue $\mathrm{T}^{2}$, Shibahara $\mathrm{T}^{3}$, Tanaka $\mathrm{Y}^{4}$, Shimono $\mathbf{M}^{1}$

${ }^{1}$ Department of Pathology, ${ }^{2}$ Department of Clinical Pathophysiology, ${ }^{3}$ Department of Oral \& Maxillofacial Surgery, 4Ichikawa General Hospital, Tokyo Dental College

We report a rare case of neurilemmoma in floor of mouth mimicking salivary gland tumor clinically. The tumor occurred at the left floor of mouth in a 27 -year-old woman. The lesion was suspected of salivary gland tumor in sublingual gland, and fine needle aspiration cytology was carried out. Cytological examination revealed long-spindle cells without findings of mitosis and hyperchromatic nuclei, and palisading suggesting neurilemmoma was seen histopathologically. Immunohistochemically, the tumor cells were positive for S-100 protein and vimentin, but negative for p63 and alpha smooth muscle actin. The tumor was finally diagnosed as neurilemmoma. We discussed differential diagnosis to other salivary gland tumors cytologically.

\section{P-30. Immunohistochemical profiles of granular cell tumors with muscular extension}

Iwahashi T, Ohno J, Ozasa R, Enoki N, Okamura K, Taniguchi K

Division of Pathology,Department of Morphological Biology, Fukuoka Dental College

Granular cell tumor is a benign neoplasm of neural crest origin that arises from soft tissues at different body sites. The tumor nests frequently spread into adjacent muscular tissues. Recurrence or metastasis is known to occur in some instances, although malignant variant is extremely rare. We examined immunohistochemical profiles and the existence of muscular extension of 3 oral granular cell tumors. Two cases showed extension into muscular tissues. All cases were positive for S-100, vimentin, NSE and CD68, while two cases with muscular extension were postive for calretinin, a calcium-binding protein.

P-31. A Case of multiplemyeloma-related amyloidosis presenting with multiple nodules in the tongue dorsum

Ioka Y, Jinbu Y, Kusama M

Department of Dentistry, Oral and Maxillofacial Surgery, Jichi Medical University

We report a case of amyloidosis, with an unusual multiple noduler in the tongue dorsum. A 76-year-old man had been suffering from multiplemyeloma and treated with molecular target therapy. The patient presented multiple nodules with erosion on the tongue dorsum. Clinical diagnosis was viral infection of the tongue, however, incisional biopsy of the tongue confirmed amyloid deposition within the tissues with evidence of erosion. Generally, amyloid deposition in the oral cavity usually involves tongue margin inducing macroglossia. The present case showed multiple nodules with erosion on the tongue dorsum, so this patient must be an unusual case of oral amyloidosis.

\section{P-32. A case of IgG4 sclerosing lesion with gingival hyperplasia as a prominent symptom}

Hatakeyama $\mathrm{S}^{1}$, Sato $\mathrm{J}^{1}$, Yodogawa $\mathrm{S}^{2}$, Ohnishi $\mathrm{T}^{2}$, Kaku $\mathrm{T}^{1}$, Nagayasu $\mathrm{H}^{2}$, Abiko $\mathrm{Y}^{1,3}$

${ }^{I}$ Graduate School of Dentistry, Health Sciences University of Hokkaido

${ }^{2}$ Department of Oral Surgery, Institute of Personalized Medical Science,Health Sciences University of Hokkaido

${ }^{3}$ Department of Dental Science, Institute of Personalized Medical Science,Health Sciences University of Hokkaido

We report a case of IgG4 sclerosing lesion with gingival hyperplasia. A 37-year-old Japanese man was admitted to our hospital with a chief complaint of gingival swelling. The diffuse gingival swelling was present in both maxilla and mandible in bad oral condition. Biopsy revealed fibrous connective tissue with a severe plasma cell infiltration. Immunohistochemical staining for IgG4 was positive in approximately $80 \%$ of the plasma cells. Blood test revealed abnormally high IgG4 level. After gingivectomy, a cleaning of the oral cavity maintained healthy gingiva. Poor dental hygiene may cause gingival hyperplasia in the patient with IgG4 related sclerosing lesion. 
P-33. A case of pseudogout of the temporomandibular joint

Ishikawa $\mathrm{A}^{1}$, Yamashita $\mathrm{Y}^{2}$, Takahashi $\mathrm{T}^{2}$, Matsuo $\mathrm{K}^{1}$

${ }^{1}$ Division of Oral Pathology, Department of Biosciences, Science of Health Improvement, Kyushu Dental College

${ }^{2}$ Division of Oral and Maxillofacial Reconstruction Surgery, Department of Oral and Maxillofacial Surgery, Science of Physical Functions, Kyushu Dental College

Pseudogout is disease in which calcium pyrophosphate dihydrate (CPPD) crystals deposit in the joint or soft tissue. The occurrence in the temporomandibular joint (TMJ) is rare. We report a case of pseudogout in the right TMJ of a 60 -year-old man presenting with the chief complaint of pain and lockjaw. His blood uric acid levels were normal. During surgery, numerous small chalky-white calcific masses were observed in the TMJ cavity. Microscopically, nodular aggregates of crystalline material with foreign-body giantcell reaction were evident. Following surgery, improvement of the lockjaw was seen, and the three-month post-operative follow-up showed good response to treatment.

\section{P-34. A case of acquired hemophilia diagnosed incidentally on stomatitis \\ Kato $\mathrm{H}^{1,2}$, Imai $\mathrm{Y}^{2}$ \\ ${ }^{1}$ Department of Dental and Oral Surgery, Motojima general Hospital. \\ ${ }^{2}$ Department of Oral and Maxillofacial Surgery, Dokkyo University of Medicine}

Acquired hemophilia is a rare acquired disease by decreased coagulant activity of factor VIII.

We report a case of acquired hemophilia diagnosed incidentally on stomtitis. A 72-years old man was referred to our hospital because of stomatitis. We suspected that the disease is some autoimmune diseases, but we could not diagnose. Because of gastrointestinal bleeding, we performed the examination of coagulation factor. Laboratory evaluation revealed a prolonged activated partial thromboplastin time, decreased coagulant activity factor VIII, and the presence of factor VIII inhibitors. Finally, we diagnosed the disease as acquired hemophilia. And steroid therapy resulted in the resolution.

\section{P-35. The effects of Malassez' epithelial rest cells to the periodontal ligament cells -findings by co-culture system-}

Matsuzaka $\mathrm{K}^{1}$, Kokubu $\mathrm{E}^{2}$, Inoue $\mathrm{T}^{1}$

${ }^{1}$ Oral Health Science Center hrc7, Department of Clinical Pathophysiology, Tokyo Dental College.

${ }^{2}$ Oral Health Science Center hrc7, Department of Microbiology, Tokyo Dental College

The purpose of this study is to evaluate the mechanism of Malassez' epithelial rest cells (MER) to periodontal ligament cells (PDL). Co-culture with MER and PDL from porcine were carried out in this study. After 5 days of co-culture, osteocalcin, bone sialoprotein (BSP), RANKL and osteoprotegrin mRNAs and alkaline phosphatase activity of PDL were analyzed. Osteocalcin, BSP and osteoprotegrin mRNAs in cocultured PDL expressed lower than those in control, but RANKL mRNA in PDL expressed higher than that in control. These results revealed that one of the MER functions play a role for maintaining the over mineralization and demineralized abilities.

P-36. Salivary glands as the source of plasma BDNF in stressed rats engaged in biting behavior Saruta $\mathbf{J}^{1,3}$, Kondo $\mathrm{Y}^{2,3}$, Sato $\mathrm{S}^{1}$, Tsukinoki $\mathrm{K}^{2,3}$

${ }^{1}$ Department of Craniofacial Growth and Development Dentistry, Division of Orthodontics, Kanagawa Dental College ${ }^{2}$ Department of Maxillofacial Diagnostic Science, Division of Pathology, Kanagawa Dental College

${ }^{3}$ Department of Maxillofacial Diagnostic Science, Division of Pathology, Research Institute of Salivary and Health Medicine, Kanagawa Dental College

We investigated whether the plasma BDNF concentration is influenced by biting. Two hours of acute immobilization stress significantly increased the amount of BDNF mRNA within the rat submandibular glands. Moreover, allowing biting behavior for the second half of the 2-h stress exposure significantly increased the amount of salivary gland BDNF mRNA relative to stress alone. Similar results were found with plasma BDNF concentrations under the same conditions. Increased BDNF, mRNA and protein expressions were observed in salivary duct cells as a result of immobilization stress and biting behavior.

P-37. Development and practical use of web presentation system for education on oral pathology Yoshida $\mathrm{W}^{1,2}$, Kubo $\mathrm{K}^{1,2}$, Sugita $\mathrm{Y}^{1,2}$, Sato $\mathrm{E}^{1}$, Wada $\mathrm{A}^{1}$, Takayama $\mathrm{M}^{1}$, Jinno $\mathrm{M}^{1}$, Torii $\mathrm{R}^{1}$, Maeda $\mathrm{H}^{1,2}$

${ }^{1}$ Department of Oral Pathology, School of Dentistry, Aichi Gakuin University

${ }^{2}$ Center for Future Oral Medical Research, Aichi Gakuin University

Our educational system "Web Presentation" was developed as one of our e-learning programs, supporting program for contemporary education needs in 2007-2009, by MEXT in Japan. Students can learn through making their own presentation slides in "Web Presentation". The slides are constructed with pictures, movies and sound material prepared by teachers or themselves. Slides are presented to other students and teachers on web. We use this system in oral pathology for the third grade of school of dentistry. It is suggested that our Web Presentation System are useful education item 